\title{
UNA ADMINISTRACIÓN PÚBLICA ADISCIPLINARIA PARA UNA SOCIEDAD DIVERSA: REFLEXIONES HISTÓRICAS, ONTOLÓGICAS, EPISTEMOLÓGICAS Y AXIOLÓGICAS
}

\author{
Jos C. N. RaAdschelders \\ The Ohio State University, Estados Unidos \\ Universiteit Leiden, Países Bajos \\ raadschelders.1@osu.edu
}

\section{RESUMEN}

Este artículo busca responder la pregunta ¿cuál es la naturaleza del estudio de la administración pública? Para ello, la primera sección explora el desarrollo histórico del gobierno y del estudio de la administración pública. Sobre esa base, en la sección segunda giro hacia el estudio de la administración pública en su contexto académico contemporáneo, al igual que muestro que su estatus se evalúa de manera diferente dependiendo del uso de definiciones más amplias o estrechas de conocimiento. La sección tercera aborda cómo estas perspectivas determinan si la administración pública puede ser considerada una disciplina en el espíritu del siglo XIX y, de no ser así, cuál(es) podría(n) ser la(s) alternativa(s). Sobre esta base se exploran los modos operativos disciplinarios, interdisciplinarios y adisciplinarios de, para y en el estudio, es decir, una exploración acerca de cuáles son los desafíos de éste, cuando se consideran como un esfuerzo disciplinario, interdisciplinario y adisciplinario. Después de esto, la sección cuarta esboza brevemente algunas de las interdependencias habitualmente olvidadas entre los fundamentos ontológicos, epistemológicos y axiológicos del estudio. A partir de este argumento, la sección quinta abordará la interrogante acerca de qué es el estudio de la administración pública. La sección final presenta un desafío para quien quiera romper el esquema de investigación de "resolver acertijos" y desee aportar algo de valor en bien de la sociedad.

Palabras clave: Teoría de la administración pública, Epistemología, Pensamiento científico, Disciplinas científicas. 


\title{
AN A-DISCIPLINARY PUBLIC ADMINISTRATION FOR A DIVERSE SOCIETY: \\ HISTORICAL, ONTOLOGICAL, EPISTEMOLOGICAL AND AXIOLOGICAL REFLECTIONS
}

\begin{abstract}
This article aims to answer the question what is the nature of the study of public administration? The first section explores the historical development of government, as well as the study of public administration. Upon that basis, I turn in section two to the study of public administration in its contemporary academic setting, as well as show that its status is evaluated differently depending upon narrower and broader definitions of scholarship. In section three, I will discuss how these perspectives determine whether public administration can be regarded as a discipline in the nineteenth century sense, and if not, what the alternative(s) could be. Upon this basis the disciplinary, interdisciplinary, and a-disciplinary modes of operation of, for, and in the study are explored- that is, an exploration of what the study's challenges are when regarded as a disciplinary, as an interdisciplinary, and as an a-disciplinary endeavor. Once that is done, I will briefly outline in section four some of the generally neglected interdependencies among ontological, epistemological, and axiological foundations of the study. Upon this argument, the fifth section is devoted to the question of what the study of public administration is. The concluding section presents a challenge to anyone who seeks to break out of the mold of "puzzle-solving" research and wishes to contribute something of value to the good society.
\end{abstract}

Keywords: Public administration theory, Epistemology, Scientific thinking, Scientific disciplines. 
La base materialista de la ciencia ha centrado su atención en los objetos más que en los valores (Whitehead 1925: 202).

La educación tiene dos propósitos: por un lado, formar la inteligencia y, por otro, formar a los ciudadanos. Los atenienses se concentraron en el primer aspecto y los espartanos en el segundo. Si bien, los espartanos vencieron, son los atenienses a quienes más recordamos (Russell 1962 [1931]: 243).

Uno de los principales obstáculos prácticos para el desarrollo de la investigación social es la división que existe entre los fenómenos sociales en una serie de campos bien definidos y supuestamente independientes que no interactúan entre sí, y en las diferentes áreas del conocimiento, como por ejemplo economí, politica, jurisprudencia, moral, antropología, entre otras. Es legitimo plantear que existe una necesidad urgente de romper estas barreras conceptuales con el fin de promover la producción cruzada de ideas [...] (Dewey 1938: 508).

[...] las ciencias sociales habian sido monopolizadas por aquellos más interesados en el descubrimiento de leyes que en el bienestar de la sociedad (Commager 1950: 205).

Un campo disciplinario difícilmente puede alcanzar el sofisticado nivel de conocimeinto digno de la formación de postgrado, si no es capaz de desarrollar de forma crítica sus fundamentos epistemológicos desde dentro de sí mismo (Ramos 1981: 102).

Estamos comenzando a reconocer que Dios no creó el universo de acuerdo a la estructuración de departamentos de nuestras universidades de investigación (Armstrong, en

Honan 1994).

\section{INTRODUCCIÓN*}

El estudio de la administración pública sirve tanto a las audiencias académica y del servicio civil de carrera, a aquellos que pertenecen a organizaciones no gubernamentales sin fines de lucro, al sector privado y también a los ciudadanos.

Al parecer, sobre todo a contar de la década de 1970, el estudio ha sido muy exitoso cuando se lo mide en términos del aumento de la producción de investigación y, por lo tanto, de las publicaciones generales y especializadas,

* Este texto es una traducción del discurso leído el I 8 de junio de 20I2, con motivo del nombramiento del autor como Profesor Titular en la Universiteit Leiden, Países Bajos. Una versión anterior, más corta y bastante diferente de este artículo fue publicada en el número de noviembre-diciembre de Public Administration Review (20I I). Algunos elementos de este trabajo han sido presentados en el John Glenn College of Public Affairs de The Ohio State University (septiembre de 20 I I) y en la Maxwell School for Citizenship and Public Affairs de Syracuse University (octubre de 20II). Me he beneficiado enormemente de los aportes de varios colegas, pero principalmente de los amables, pacientes y cuidadosos comentarios sobre repeticiones en el texto por parte de mi colega en el Glenn College, profesor Anand Desai y de la profesora Tina Nabatchi, una colega de la Maxwell School. Finalmente, la versión en inglés de este trabajo fue editada por Travis Whetsell, estudiante del programa de doctorado en el Glenn College, quien es el editor ejecutivo de Armed Forces \& Society. La versión holandesa ha sido cuidadosamente editada por la Sra. Wilma der Weduwe-de Groot, JD, cuya formación y experiencia como abogada y jueza le han dado excelentes capacidades en la lengua holandesa, las cuales yo carezco. 
de programas independientes de administración pública y, por ende, del cuerpo académico de administración pública; y de la cantidad de trabajo financiado y consultorías realizadas por académicos, continuando así la conexión entre el mundo académico y la práctica cotidiana. En cada década, y debido al auge de este estudio, algunos han querido hacer un inventario del estado del arte de la investigación en administración pública. Todo esto, en un intento por categorizar las tendencias y determinar hacia dónde se podría dirigir el estudio en un futuro (cercano) y en qué ámbitos temáticos se podrían y/o deberían ampliar los esfuerzos en investigación (para una reseña general de esta bibliografía y sus tendencias durante la última década, ver Raadschelders y Lee 2011).

El mundo académico de la administración pública ha construido una impresionante estructura de conocimiento, tanto sobre la base de investigación original y en la búsqueda de información en base a las contribuciones de otros estudios y disciplinas, en la medida que sean relevantes para comprender el gobierno. No obstante, pareciera limitarse a extrapolar el cómo se podría desarrollar el estudio a partir de intereses temáticos recientes, actuales e incluso, quizás, temas de moda. Sin embargo, correspondería a cualquier estudio que ha madurado en términos de productividad, personas y programas, explorar las bases y supuestos sobre los que se basa su conocimiento, de forma tal que se pueda trazar un curso hacia el futuro, donde el conocimiento temático -generado mediante la investigación y difundido a través de la enseñanza- esté inmerso en cimientos explícitos.

El estudio descansa en, al menos, cuatro cimientos, de los cuales ninguno recibe suficiente atención. Para comenzar, necesitamos prestar mayor atención a la filosofía en el estudio de la administración pública ${ }^{1}$ (Van Braam 1989), de suerte tal que sus egresados, la mayoría de los cuales harán carrera fuera del mundo académico, puedan reconocer los fundamentos y fuentes del conocimiento de ese estudio. En segundo lugar, los académicos que imparten docencia en la carrera de administración pública también necesitan prestar mayor atención a la filosofía de gobierno (Dimock 1958), de modo que sus egresados tengan una comprensión de lo que significa gobierno, que los impulse más allá de ser meros tecnócratas que busquen dentro de sus herramientas la que le sea de mayor utilidad. Cualquier programa de asuntos públicos debe informar a los estudiantes de los valores y fundamentos bajo las cuales operan sus gobiernos. Una ciudadanía y un cuerpo de funcionarios públicos informados son aquellos

1 Al hablar del estudio, utilizo "administración pública"; al hablar del mundo de los profesionales públicos, utilizo "gobierno". 
que acogen, y no rehúyen, las consideraciones con carga valórica. Tercero, se debe procurar inculcar la comprensión de cómo los diversos fundamentos y perspectivas en una filosofía del estudio tributan a los fundamentos y perspectivas del gobierno en la sociedad, y viceversa (Hodgkinson 1982). En efecto, no podríamos afirmar que la generación de conocimiento en el mundo académico está exenta de valores y que este conocimiento, a su vez, no ejerce influencia sobre los valores subyacentes en la toma de decisiones del sector público y formulación de políticas. Por el contrario, ¿podrían los académicos sinceramente afirmar que su investigación no está influenciada en absoluto por valores existentes en el gobierno y la sociedad? En cuarto y último lugar, necesitamos prestar mayor atención al impacto que la historia (como actividad de registro) y el pasado ejercen sobre la forma en que teorizamos, actuamos y respondemos a los desafíos de hoy (Raadschelders 1998a 2010b). El primer elemento que se destacará en este artículo es "una filosofía de la administración pública", y también se prestará atención a consideraciones normativas. Cabe destacar que es sobre estos cuatro fundamentos que suele en general descansar la investigación. Sin embargo, mientras la mayor parte del esfuerzo se concentra en los tecnicismos de la investigación (más aún, primordialmente en el trabajo lógico-empírico), estas bases han quedado relegadas a un segundo plano. ¿Por qué ocurre esto?

Gran parte de la investigación académica del estudio se centra en entregar comprensión y soluciones a problemas prácticos a través de investigación empírica basada en evidencias, lo que deja mucho menos tiempo disponible para reflexionar acerca de la naturaleza del gobierno y su estudio. Esto resulta interesante por dos motivos: 1) a contar de la década de 1960 ha ido disminuyendo la confianza en las instituciones gubernamentales; y 2) persiste un desacuerdo sobre el estatus académico de la administración pública. En el presente artículo me centraré principalmente en lo segundo, pero mencionaré brevemente el porqué esto es importante para comprender el declive de la confianza en el gobierno, y cómo el estudio puede ayudar a los profesionales a responder a las demandas ciudadanas.

Algunos académicos consideran que el estudio carece de disciplina, la que se debería adquirir mediante el rigor metodológico; otros sostienen que el estudio atiende a un mundo real que no se puede capturar de forma adecuada con un enfoque determinado. Se pueden concebir varias razones de porqué existe poco o casi nada de reflexión en torno a la naturaleza del estudio. En primer lugar, esto se podría deber a que muchos investigadores en administración pública exploran problemas sociales y desafíos gubernamentales, que aparentemente requieren de atención y solución inmediatas. Su argumento se basa en que la investigación empírica basada 
en evidencias que, a fin de cuentas, hacen la diferencia en el mundo real, es más valiosa que las reflexiones acerca de la naturaleza del conocimiento en el estudio. Incluso se asume implícitamente que la búsqueda de la investigación basada en evidencia dará lugar a una identidad académica más clara. Existen varias otras razones que, en efecto, no se limitan a los investigadores en administración pública, sino que a los cientistas sociales en general, en particular aquellos formados a contar de la década de 1980 .

En segundo lugar, muchos cientistas sociales se ocupan principalmente del presente, se desentienden del pasado y desarrollan poca sensibilidad hacia la manera en que su investigación encaja en un contexto que trasciende el área temática de su trabajo. Es decir, su trabajo está más impulsado por la necesidad de posicionarse en la literatura relevante y contemporánea que por el esfuerzo de ubicarlo en un tiempo y contexto. Por otra parte, muchos resultados de investigación son presentados como generalizaciones, sin considerar en absoluto las circunstancias específicas (tiempo y contexto) en las cuales se llevaron a cabo dichas investigaciones. Aunque su objetivo es replicar la investigación en diferentes entornos geográficos, organizacionales y culturales, las observaciones acerca de las limitaciones de los hallazgos en las secciones finales y de discusión de artículos de revistas son, a menudo, superficiales y esperables.

En tercer lugar, muchos académicos no están entrenados para explicar su propia comprensión acerca de la realidad que investigan. Trabajan con hipótesis y conjuntos de datos que -aunque bien generados- están desconectados de una comprensión específica de la realidad. Por ejemplo, la diferencia entre creer que la realidad puede ser conocida de manera objetiva, por un lado, y por otro, la creencia de que esta es una construcción social, tiene consecuencias significativas para determinar qué podemos conocer y cómo podemos hacerlo (esto se discutirá con más detalle a continuación), y asimismo, cómo y qué podemos considerar posible con respecto a remediar, mejorar, alterar, adaptar $-\mathrm{y}$ así sucesivamente- una situación o problema específico.

Esta tercera consideración nos conduce a la cuarta, el hecho que muchos académicos no indagan en qué y cómo pueden conocer. Juegan con datos y muestran gran sofisticación en los modelos de análisis cuantitativos, pero están menos preparados para explicar si es que los datos en realidad proporcionan una representación adecuada de la realidad que buscan describir. En efecto, muchos cientistas sociales describen la realidad en términos fácticos, pero sin prestar atención explícita a cómo se establecieron dichos hechos. ¿QQuién decide qué investigar, qué datos recopilar? Por otra parte, cualquier consideración acerca de las bases y marcos del conocimiento 
pareciera estar relegada a la filosofía, creando así -como los llamaba Kantexpertos científicos que poseen "erudición ciclópea" pero les falta el ojo de la filosofía (1988: 50). Kant observa que "Nadie se ocupa de la sabiduría, porque convierte a la ciencia -que es un instrumento de la vanidad-en algo bastante pequeńo" (1996: 28, nota 30).

En quinto y último lugar, aquellos cientistas sociales que consideran que la realidad puede ser conocida de forma objetiva, también creen que su conocimiento solo es científico, cuando se percibe la realidad social como una serie de hechos que están separados de los valores. Esta separación valor-hecho no es solo un tema (y posiblemente un problema) en el mundo académico, sino también un desafío en el mundo profesional, donde -en lo que respecta a los funcionarios públicos de carrera- las políticas y decisiones son presentadas y legitimadas ante el público como si estuviesen basadas en hechos recopilados de forma científica, por expertos con formación científica, excluyendo implícitamente a gran parte de la ciudadanía de una participación sustantiva, más allá de una audiencia o de una consulta popular requerida. No obstante, al saber que los ciudadanos expresan una mayor satisfacción con la prestación de servicios públicos cuando su consumo es por elección propia más que por coerción (Brown 2007: 568), también podemos suponer con certeza que en muchos casos la calidad de la formulación de políticas no se beneficia solo al incluir a los ciudadanos (Lindblom 1990), sino también al ser abierta y franca en cuanto a los valores que sustentan las decisiones que son, a fin de cuentas, políticas ${ }^{2}$. Hegel caracterizaba a los funcionarios públicos como la nueva clase universal, los nuevos guardianes de la democracia cuyo consejo experto consiste en asesorar al gobernante (Hegel 1991, par. 287-303, véase también Brooks 2006). Esto ya no es aceptable en una sociedad más alfabetizada. En cambio, los funcionarios públicos ahora asesoran tanto a gobernantes como ciudadanos y en el desempeño de ese papel podrían, y quizás incluso deberían, ser convocados para delinear las justificaciones para las decisiones políticas.

En las palabras de Yates:

Si los burócratas no iluminan, analizan y educan a los ciudadanos acerca de los conflictos de valores, ¿qué otras instituciones lo harán? Simplemente [...] es evidente que no se encuentra en el procedimiento operativo estándar de otras instituciones políticas

2 En una nota al margen: algunas políticas o acciones podrían no beneficiarse de los aportes ciudadanos, en particular cuando se refieren a aspectos no contenciosos (Irvin y Stansbury 2004: 62). 
principales el desempeñar este papel normativo (1981: 46).

Lo que resta del presente texto está centrado en la interrogante de qué debemos hacer para trazar un futuro para el estudio, que sea lo suficientemente amplio tanto para el profesional como el académico y que tenga, por otro lado, una clara identidad académica. Esta pregunta no puede sino estar precedida por otra -¿cuál es la naturaleza del estudio de la administración pública?- la que se volvió cada vez más apremiante en la medida que se cuestionó su identidad académica a contar de la década de 1960 por estar en una suerte de crisis. Se han sugerido a lo menos tres soluciones a esta crisis de identidad: 1) organizar el estudio en torno a un concepto central; 2) promover el rigor metodológico (es decir, el conocimiento basado en la ciencia, en estricto sentido; véase a continuación); o 3) convertirse en una escuela profesional. No obstante, ninguna generó consecuencias significativas. ¿Podría ser que se debería buscar una solución a la supuesta crisis de identidad de la administración pública más bien en consideraciones históricas, ontológicas, epistemológicas y axiológicas? Estas cuatro categorías de consideraciones deberían ayudar a definir los cimientos profundos del estudio y, por ende, revelar con qué fundamentos se puede afirmar que la administración pública es un estudio maduro, aunque a veces adisciplinario.

A los ojos de muchos investigadores en administración pública, el estudio sufre de una crisis de identidad, a tal punto, que un colega incluso ha afirmado que al compararlo con la adolescencia, esta parece idílica (Rhodes et al. 1995: 1)33. ¿Por qué llegó a ser tan apremiante esta pregunta, acerca de la naturaleza académica y estatus del estudio? Una respuesta lógica exige que primero retrocedamos en el tiempo y observemos brevemente el desarrollo del gobierno por sí mismo, además de ver cómo la aparición y expansión éste promovió el surgimiento del estudio de la administración pública (véase la primera sección).

La primera sección explora el desarrollo histórico del gobierno y del estudio de la administración pública. Sobre esa base, en la sección segunda giro hacia el estudio de la administración pública en su contexto académico contemporáneo, al igual que muestro que su estatus se evalúa de manera diferente dependiendo del uso de definiciones más amplias o estrechas de conocimiento. La sección tercera aborda cómo estas perspectivas determinan si la administración pública puede ser considerada una

3 El concepto de crisis de identidad fue acuñado primero por el psicólogo del desarrollo Erik H. Erikson (1968), y se refiere al período durante la adolescencia en que los púberes y jóvenes adultos están en búsqueda de su identidad. 
disciplina en el espíritu del siglo XIX y, de no ser así, cuál(es) podría(n) ser la(s) alternativa(s). Sobre esta base se exploran los modos operativos disciplinarios, interdisciplinarios y adisciplinarios de, para y en el estudio, es decir, una exploración acerca de cuáles son los desafíos de éste, cuando se consideran como un esfuerzo disciplinario, interdisciplinario y adisciplinario. Después de esto, la sección cuarta esbozará brevemente algunas de las interdependencias habitualmente olvidadas entre los fundamentos ontológicos, epistemológicos y axiológicos del estudio. A partir de este argumento, la sección quinta abordará la interrogante acerca de qué es el estudio de la administración pública. La sección final presenta un desafío para quien quiera romper el esquema de investigación de "resolver acertijos" y desee aportar algo de valor en bien de la sociedad.

\section{ALGUNAS REFLEXIONES HISTÓRICAS ACERCA DEL SURGIMIENTO DEL GOBIERNO Y DE SU ESTUDIO}

Ningún ser humano ha vivido en completo aislamiento. Las personas siempre han habitado en grupos de tamaño variable. En los grupos pequeños, de, por ejemplo 30-50 personas hasta incluso algunos cientos, los problemas comunitarios se podían resolver sobre la base de relaciones de amistad y parentesco, tanto de forma diacrónica como intergeneracional. Los biólogos evolucionistas supusieron que el altruismo recíproco opera a lo largo del tiempo. No obstante, tan pronto como las sociedades comienzan a abarcar miles de personas o más, las relaciones de amistad y parentesco se transforman en una base demasiado débil para resolver los problemas que conciernen a la comunidad completa. Es en este punto que las personas, mediante un mecanismo de ensayo y error (hasta donde se conoce), desarrollan estructuras de autoridad y de toma de decisiones que son percibidas como legítimas por todos, incluso cuando ninguna persona puede afirmar que conoce a todos los demás. Es entonces cuando la comunidad imaginaria sustituye a la comunidad física; es en este punto donde se crea y establece el gobierno. Es decir, el gobierno surge desde dentro de las sociedades sedentarias primarias y se expande mediante, por ejemplo, el aumento de la división del trabajo y de la densidad poblacional.

Mirando la historia del gobierno, pareciese que éste avanzó del surgimiento al diseño. Los gobiernos surgieron $y$, tan pronto las personas se hicieron conscientes de su propia existencia, pudieron ser objetos de un diseño intencional. Durante la mayor parte de la historia, los gobiernos simplemente se manifestaban, a pesar que hubo momentos en que las personas reflexionaban acerca del mejor tipo de acción colectiva legitimada dentro de comunidades imaginadas. Estas reflexiones a menudo tomaron un formato utópico, por ejemplo en las consideraciones 
acerca del gobierno ideal. No obstante, de vez en cuando y a medida que transcurría el tiempo, las personas descubrieron que dichas reflexiones en realidad se podían materializar; que el gobierno no era solo el producto de un caprichoso gobernante y del comportamiento de la élite, sino que efectivamente podía ser el resultado de un diseño intencional. La República de los Siete Países Bajos Unidos es un ejemplo de un sistema de gobierno donde se revelaron elementos de surgimiento (las provincias medievales, matrimonios burgundios que los unificaron) y un poco de diseño (considérese la Unión de Utrecht que estableció una confederación en 1579 y la decisión de no reconocer al monarca español como soberano en 1581). Las élites en la República de los Países Bajos no estaban realmente interesadas en transformar las estructuras institucionales existentes en las distintas provincias. Otro ejemplo de la naturaleza complementaria entre surgimiento y diseño es el de los Estados Unidos de América, cuyas colonias surgieron durante el transcurso de más de 150 años, donde en las décadas de 1770 a 1780 los Padres Fundadores designaron una nueva estructura gubernamental que aún sigue en pie.

Si deseamos comprender los desafíos que enfrentan los gobernantes actuales, necesitamos evaluar esta relación entre surgimiento, por un lado, y diseño, por otro. Durante la mayor parte de la historia, los gobiernos surgieron y cambiaron con el tiempo. En algunas ocasiones, estos cambios fueron el resultado de reformas conscientes, tales como aquellas introducidas por el Rey Šulgi (2094-2047 A. C., cronología media) de la Tercera Dinastía de Ur (2114-2004 A. C.), el estadista chino Shang Yang (390-338 A. C.), el cual ayudó a forjar el camino para la dinastía Qin, y Napoleón, cuyas reformas en realidad dejaron huellas por todo el mundo, a pesar que apenas estamos conscientes de ello. A menudo las reformas fueron impuestas desde el exterior (ver las ocupaciones napoleónicas y germánicas en Europa Occidental), pero adaptadas a las circunstancias locales una vez que el país ocupante se había retirado (Wunder 1995, Rugge 2000). Desde los inicios de la era moderna, varios países europeos colonizaron gran parte del mundo e impusieron sus estructuras gubernamentales por sobre los acuerdos gubernamentales locales que imperaban. Esto ha generado mucho daño, particularmente en África, dado que las nuevas fronteras estatales negociadas literalmente atravesaban las zonas tribales y, en algunos casos, simplemente desplazaron las supremacías existentes con fronteras reconocidas (Davidson 1982). Actualmente no existe masa de tierra en el mundo, a excepción de la Antártida ${ }^{4}$, que no forme parte de

4 La Antártida, por cierto, está sujeta a la gobernanza a través de un vasto sistema de normas internacionales. El Sistema del Tratado Antártico define tareas y responsabilidades para un amplio espectro de actores. 
un Estado territorial que funcione a través de la burocracia.

En otras palabras, el gobierno como estructura institucional formal surge en aquellas sociedades sedentarias donde el tamaño de la población impide la interacción cara a cara entre todas las personas. Es inevitable que surja el gobierno en comunidades imaginadas. ¿Cómo se puede comprender semejante estructura institucional? Supongamos que el gobierno es un caso especial de gobernanza. Gobierno, por lo general, se refiere a aquel grupo de instituciones y organizaciones donde la soberanía es investida, y cuya autoridad se expresa en el hecho que es el único actor que: 1) puede tomar decisiones vinculantes en nombre de toda la población; y 2) tiene el derecho a usar la fuerza con el objeto de mantener la ley y el orden ${ }^{5}$. Gobernanza se refiere a todas aquellas instituciones y organizaciones que, de alguna manera, contribuyen a dirigir la sociedad. La relación entre gobernanza y gobierno se puede conceptualizar como parte de una doble hélice que consiste en los deseos y capacidades asociativas genéticamente grabadas de las personas, por un lado, y en las estructuras institucionales formales, por el otro (gobernanza y gobierno). En las sociedades pequeñas, la asociación se expresa a través de relaciones de parentesco y amistad por sí solas, y es suficiente para enfrentar problemas de naturaleza colectiva. En las comunidades imaginadas, la asociación no puede sino expresarse en términos de ciudadanía, puesto que las relaciones de amistad y parentesco ya no pueden unir por sí solas a todas las personas.

¿Es esta pequeña incursión en la historia administrativa de alguna manera relevante y/o necesaria para la comprensión del gobierno hoy en día? Muchas investigaciones se concentran en el aquí y el ahora, pero la administración y el gobierno han existido desde mucho antes de que las personas efectivamente comenzaran a registrar sus acciones (por lo general, transacciones económicas). La noción de que el surgimiento y desarrollo del gobierno promovieron el estudio de la administración pública nos conduce automáticamente a la conclusión de que, con el fin de concebir cómo puede o debería ser el futuro del estudio, éste tiene que estar ligado a: 1) una profunda comprensión de los orígenes y posterior desarrollo del gobierno; 2) una profunda sensibilidad respecto a la naturaleza de los

5 Con frecuencia, las definiciones de Estado se centran en el uso legítimo de la coerción o fuerza, un énfasis weberiano que a menudo es aceptado (por ejemplo, Tilly 1975, Dyson 1980). No obstante, el uso legítimo de la fuerza es solo un ejemplo de una categoría más amplia, a saber, de las decisiones vinculantes. Por otra parte, y sobre todo en el mundo contemporáneo, existen muy pocas sociedades donde los gobiernos aún comparten la autoridad para tomar decisiones vinculantes para todos los ciudadanos con otras asociaciones sociales (como en el pasado, la religión organizada). 
desafíos actuales que enfrenta la sociedad y que en cierta medida se espera que aborde el gobierno y, por supuesto; 3) lo que a nosotros, la ciudadanía en general, nos gustaría que sea una sociedad justa y su gobierno, y cómo llegar hasta ese punto ${ }^{6}$. Se puede adquirir una comprensión profunda acerca de la presencia del pasado en términos administrativos (Fesler 1982) a través del estudio de la historia administrativa pero, habiendo escrito acerca de esto en diversos medios, no resulta necesario reiterar los argumentos acerca de por qué esto es un esfuerzo productivo (Raadschelders 1998a, 2010b). Retomaré al final de este artículo temas que conciernen a los desafíos sociales y gubernamentales de hoy, así como también la sociedad justa.

El estudio de la administración pública se centra en las múltiples maneras en las cuales las personas de las sociedades sedentarias y densamente pobladas logran equilibrar su deseo natural por una asociación a pequeña escala con la necesidad artificial de estructuras institucionales formales (a gran escala). Por lo tanto, el estudio de la administración pública no se refiere únicamente al liderazgo, las relaciones intergubernamentales, la organización y gestión, la formulación de políticas, los instrumentos de políticas, la planificación, presupuesto y finanzas, además de la gestión de recursos humanos, todos vinculados a la estructura y funcionamiento del gobierno en sí mismo (el principal foco de estudios hasta la década de 1960). Trata también el estudio de las maneras en que las entidades sociales (no tan solo organizaciones no gubernamentales, sino también iglesias, sindicatos, clubes deportivos, juntas vecinales, sistemas de gestión de recursos comunes, entre otros) y la ciudadanía participativa (significativa) contribuyen a la gobernanza de la sociedad. En efecto, desde la década de 1960 el estudio de la administración pública ha ampliado su ámbito de manera significativa, tanto en lo que respecta al estudio de los tipos de estructuras de las instituciones y de asociaciones (incluyendo de forma especial los recursos comunes y las organizaciones sin fines de lucro), como en términos de interés temático (por ejemplo, ética y valores dentro del sector público, motivación del sector público, terrorismo, gestión de emergencias, administración electoral, gobierno electrónico, creación de

6 Lo que los ciudadanos desean que sea una sociedad justa, incluye el deseo de un buen gobierno. La disminución de la confianza en el gobierno, como ha sido denunciada en diversos grados, es un grave problema en la mayor parte del mundo occidental, y requiere de atención para la dinámica en las relaciones entre titulares políticos (y la manera en que estereotipan la burocracia) y los funcionarios públicos (y la manera en que la primacía de la doctrina política los mantiene en las sombras). Para los Estados Unidos, véase Public Trust in Government: 1958-2010, Centro de Investigación Pew para el Pueblo y la Prensa, publicado el 18 de abril de 2010; consultado el 3 de abril de 2012. Para una visión comparativa, véase Bouckaert (2011). 
redes y gestión participativa, y así sucesivamente).

La búsqueda de como comprender el gobierno difiere según las exigencias de cada época y contexto. En el mundo antiguo y hasta finales de la Edad Media europea, la comprensión de gobierno se centraba en el liderazgo ideal y/o la relación realista entre gobernantes y gobernados (considérese al pensador indio Arthaśāstra de Kautilya, el Fürstenspiegel en la Edad Media y El Príncipe de Maquiavelo) y en la planificación física (por ejemplo, el plan urbano ideal). Una vez que en Europa el Estado territorial, más que la ciudad-Estado o el imperio, se hubo consolidado exitosamente como el principal actor que vinculaba a las personas y ante el cual la población acudía cada vez más para la obtención de servicios (a contar del siglo XII), surgió un estudio de la administración pública con una orientación mucho más práctica, que priorizaba la formulación e implementación de políticas, los formularios y procedimientos administrativos, las descripciones de cargo para funcionarios públicos, los tipos de ordenanzas y de correspondencia oficial y así sucesivamente, pero con énfasis en la mecánica interna de dirigir un gobierno (esto es, experiencia práctica para el funcionario público). Hacia mediados y finales del siglo XVIII, esto también llegó a incluir una priorización por las políticas y servicios sustantivos de bienestar (Christiaan von Wolff. Véase Rutgers 2010).

Cómo lo concebimos hoy, el estudio de la administración pública surgió en la segunda mitad del siglo XIX, en respuesta a un gobierno en rápido crecimiento del que se esperaba que cada vez más proporcionase soluciones a los problemas causados por la industrialización (como las condiciones laborales y explotación, el trabajo infantil, la extensión de la jornada laboral), la urbanización (como la falta de planificación urbana, el hacinamiento, la falta de higiene, vecindades hacinadas) y el rápido crecimiento demográfico (lo que generaba, por ejemplo, una insuficiencia en los servicios de salud y utilidad pública). Estos tres grandes cambios ambientales crearon una demanda por un gobierno para el cual no existían precedentes históricos y con el cual, por lo tanto, no había experiencia. Súbitamente, los funcionarios públicos de todos los niveles se encontraban "sellando grietas" exigencias tan masivas y diversas (Skowronek 1982, Raadschelders 1990, Stillman 1999: 57). Los gobiernos de ambos lados del Atlántico estaban creciendo con celeridad en términos de diferenciación organizacional (Raadschelders 1997a), regulación (Page 2001), ingresos y gastos (Webber

7 Acá, "sellar grietas" se refiere a una actividad en la construcción de cabañas de troncos donde estos, una vez instalados, deben ser sellados, esto es, se debe rellenar las grietas con barro, arcilla o musgo (Stillman 1999: 57). 
y Wildavsky 1986), y tamaño del personal (Raadschelders 1994); por ello, los profesionales deseaban orientación respecto a cómo hacer frente a este nuevo fenómeno. Por consiguiente, los intereses del estudio eran muy prácticos, pero se referían a necesidades externas al gobierno (servicios para la población), y estaban definidos por profesionales a ambos lados del Atlántico ${ }^{8}$. Necesitaban conocimientos útiles acerca de los principios de gestión, liderazgo y organización, pero los querían enmarcados en un currículum mucho más amplio que prestase atención a la historia, el derecho, la economía, la política y la ética, con el fin de garantizar que los (futuros) funcionarios públicos pudiesen tener una comprensión amplia (generalista) de la sociedad a la que iban a servir (Hoffmann 2002). A contar de la década de 1930, algunos académicos abogaron por un enfoque más científico y lógico-empirista, mientras que otros continuaron considerando al estudio en su enfoque clásico de "sabiduría práctica" (que hoy en día también incluye la teoría crítica) y/o en su anterior manifestación de experiencia práctica. En las últimas décadas, el estudio ha sido enriquecido por la teoría crítica, el pragmatismo y la teoría de la acción, así como por las perspectivas relativistas o postmodernas que incluyen, entre otros, la hermenéutica, el narrativismo, el interpretativismo y la fenomenología.

En otro escrito he diferenciado cuatro enfoques intelectuales al estudio, esto es, la sabiduría práctica, la experiencia práctica, el conocimiento científico y las perspectivas relativistas, que difieren mucho entre sí (Raadschelders 2008). De hecho, son tan diferentes que pueden ser considerados como una manifestación que el estudio carece de una identidad clara. ¿Es una ciencia, un oficio, una profesión o un arte? (Lynn 1996) ¿Podría ser todas las anteriores y, en tal caso, qué significaría esto para la naturaleza y futuro del estudio? Esta pregunta es importante a la luz del enorme incremento de los programas independientes de administración pública (con independencia en términos organizacionales del derecho, los estudios organizacionales y la ciencia política) a contar de la década de 1970 en los Estados Unidos (Raadschelders 2011a: 141) y Europa Occidental (Verheijen y Connaughton 1999, 2003), a contar de la década de 1980 en India, Japón y Corea del Sur (Raadschelders 2009), y de la década de 2000 en China y otros países asiáticos, así como en diversos países africanos y

8 Por supuesto, ya existe una tradición muy práctica de enseñanza en la administración pública anterior a la industrialización en diversas partes del mundo, por ejemplo la capacitación de funcionarios públicos en la antigua China (a contar de la dinastía Han, siglo II A. C.), en la antigua India (véase Arthasastra), en Corea (donde la primera escuela comenzó hace 600 años), en Alemania desde la segunda mitad del siglo XVII, en Francia desde inicios del siglo XVIII, y en Reino Unido desde la segunda mitad del siglo XVIII (por ejemplo, el Indian Civil Service). 
latinoamericanos.

La pregunta acerca de si es un arte, un oficio, una profesión, una ciencia o todas las anteriores, será abordada en tres pasos. En primer lugar, ¿qué es característico del enfoque actual de la ciencia? Esta pregunta será abordada en la siguiente sección, que presta atención a: 1) dos definiciones bastante diferentes de "ciencia"; 2) la definición de "disciplina"; 3) el hecho que los estudios científicos supuestamente deban tener límites; 4) el tema de cómo puede percibir y se percibe la realidad; y 5) el problema de qué caracteriza a las ciencias sociales hoy en día. Esta sección general es necesaria para comprender la naturaleza específica de la administración pública.

En segundo lugar, ¿cómo sería la administración pública si fuese una disciplina? Si consideramos que nunca podría ser una disciplina, debemos determinar por qué es así. Por ende, necesitamos evaluar su carencia de límites, quién define su objeto de estudio, y si la crisis de identidad del estudio es única o común (sección tercera).

Finalmente, en la última sección, se argumenta que la administración pública es un estudio adisciplinario que afronta varios retos. El primero es que el gobierno posee características locales, regionales, nacionales e internacionales distintivas, y cada uno de estos niveles jurisdiccionales tiene tanto necesidades superpuestas como diferenciadas. Segundo, que el estudio debe conectar niveles de análisis micro y macro, y por lo tanto combinar la investigación que se basa en el conocimiento de datos de base individual con los enfoques institucionales que exploran el contexto en que los humanos inevitablemente operan. Tercero, que en un mundo académico que se nutre de la especialización y la adopta (en términos de conocimiento, método y organización), y en un mundo profesional que pregona la experiencia, el estudio debe proporcionar una perspectiva generalista, especialmente para los funcionarios públicos de carrera de nivel medio y alto (véase Hoffmann 2002) ${ }^{9}$. Cuarto, esto significa que el estudio debería continuar uniendo a profesionales y académicos, aun cuando sus diferencias de intereses podrían nunca ser subsanadas por completo. En quinto y último lugar, el estudio debería equilibrar su prioridad tanto por la investigación como por la formación. La investigación requiere tanto especialistas como generalistas, mientras que la enseñanza puede y debe proporcionar una base para la perspectiva de un generalista.

9 Waldo habló del "administrador completo" (1984 [1948]: 99). Este término también ha sido empleado por Johnson (1982). 


\section{EL ENFOQUE ACTUAL DE LA “CIENCIA”}

En el mundo angloamericano, la "ciencia" se comprende y define como una rama de estudio que observa y clasifica hechos, los que a su vez, describen, explican y predicen fenómenos naturales y (se espera) sociales, por medio de "leyes". Esta definición de la ciencia se remonta a la Ilustración del siglo XVIII, y se debe en especial a la distinción que hizo David Hume entre los hechos (el objeto de la ciencia) y los valores (el objeto de la política y la opinión pública), una segmentación que ha sido adoptada incondicionalmente por los empiristas lógicos, de quienes Herbert Simon es un excelente representante. Esta definición de ciencia es más estrecha que la noción previa al siglo XVIII, que la definía como un conjunto de conocimiento general (recogida en el alemán como Wissenschaft y en términos comparables en otras lenguas germánicas) que busca mejorar la comprensión de los fenómenos naturales y sociales ${ }^{10}$. Dwight Waldo estaba consciente de esta distinción cuando observó que la ciencia se podía definir como "[...] un conjunto de conocimiento organizado" en general (Wissenschaft) o como "determinado tipo y calidad de conocimiento y procedimiento" (es decir, ciencia) (1984: 182, nota al pie 50). En la definición amplia de ciencia como Wissenschaft, el énfasis se encuentra en la epistemología. ¿Cómo se justifican las afirmaciones de conocimiento? ¿Cómo definimos el conocimiento? ¿Cuáles son las fuentes del conocimiento? ¿Cuál es la relación entre el objeto del conocimiento y el investigador? Por otra parte, la ciencia, en su definición más exacta, está mucho más centrada en la metodología y los métodos, simplemente en función de la creencia que la única base para el conocimiento científico es la epistemología de tipo lógico-empírico ${ }^{11}$. ¿Qué métodos de análisis se pueden utilizar para sustentar el conocimiento? ¿Cómo se recopilan y analizan los datos para responder una pregunta específica? Mientras que la epistemología se centra en la filosofía de qué podemos conocer, la metodología se ocupa de las prácticas sobre cómo podemos conocer y, por

10 Wissenschaft se traduce mejor como "rama del conocimiento" y es el término utilizado en las lenguas germánicas (por ejemplo, wetenschap en holandés, vetenskap en sueco). La ciencia en su sentido más amplio, anterior al siglo XVIII, como "conjunto organizado de conocimiento", aún se utiliza en las lenguas románicas (por ejemplo, science en francés, ciencia en español, scienza en italiano) (Raadschelders 2011b: 41).

11 El término empirismo lógico es preferido por sobre aquel de positivismo, puesto que este último podría confundirse con la comprensión de positivismo de Auguste Comte. Además, si bien Simon se sentía cómodo tratando "empirismo" y "positivismo" como sinónimos (1997: 68), el positivismo o positivismo lógico en realidad es un tipo de empirismo porque otros tipos de empirismos (por ejemplo, taxonomía, tipología) no pueden ser etiquetados como positivistas (lógicos) (véase Phillips 1987: 41). 
ende, se centra en los métodos.

La epistemología lógico-empirista sostiene que lo que podemos conocer son los hechos observables; las metodologías interpretativistas, en cambio, aceptan que podemos indagar aún más (sentimientos, intuiciones, comprensiones; véase Einfühlung de Max Weber).

Los investigadores han intentado clasificar los conjuntos de conocimiento desde la Antigüedad. Aristóteles, por ejemplo, dividió las ciencias (conocimientos) en tres ramas: las ciencias teóricas orientadas a la verdad (por ejemplo, las matemáticas), las ciencias prácticas que servían para conseguir buenas acciones (por ejemplo, la medicina, la política y, podríamos agregar, la administración), y las ciencias productivas que buscaban perfeccionar las cosas (por ejemplo, la poesía y la retórica) (Mahdi 1971: 229). A contar del siglo XVII, es común distinguir entre las ciencias naturales (que estudian los fenómenos naturales), las ciencias sociales (que estudian los fenómenos sociales) y las humanidades (que estudian las expresiones creativas de la humanidad).

Dentro de cada una de estas tres ramas principales, se demarcan entre sí diversos conjuntos de conocimiento, sobre la base de su objeto de estudio. Tradicionalmente, es decir desde el siglo XIX, un conjunto de conocimiento es una disciplina cuando se caracteriza por un conjunto consistente y coherente de conceptos y teorías que explican un conjunto particular de fenómenos (es lo que Kuhn 1973 [1962] denomina un paradigma) y está claramente demarcada de otras disciplinas. Determinar qué conceptos y teorías son los más adecuados, qué investigaciones se deberían realizar y qué constituye una investigación de calidad, son el ámbito exclusivo de la comunidad de académicos en una disciplina. Las matemáticas, física, química y biología son excelentes ejemplos de disciplinas en las ciencias naturales. En las ciencias sociales, algunos ejemplos podrían incluir la psicología y economía, aunque no pueden reclamar el control exclusivo sobre las teorías e investigaciones en su "disciplina". Algunos ejemplos de disciplinas en las humanidades son las lenguas, bellas artes, historia y teología. En el mundo académico, tal como está organizado desde el siglo XIX, no se espera que un especialista en chino pueda juzgar la calidad de una pieza de bellas artes, más allá de una atracción o rechazo emotivos de la misma. La diferencia entre las ciencias naturales, por un lado, y las ciencias sociales y humanidades, por el otro, es que las primeras en realidad buscan, definen y operan sobre las regularidades y probabilidades, mientras que las restantes, en el mejor de los casos, funcionan con generalizaciones 
de atisbos legales ${ }^{12}$. Se debe tener en cuenta que la afirmación anterior se basa en un sentido de comprensión y organización de la ciencia del siglo XIX y XX, que está rápidamente quedando obsoleto con el aumento de la interdisciplinariedad.

No obstante, hasta ahora estas fronteras disciplinarias tradicionales son reforzadas por las estructuras organizacionales, como un departamento dentro de una universidad (por ejemplo, el Instituto de Administración Pública en la Facultad de Ciencias Sociales, en la Universiteit Leiden) o como una escuela independiente de las universidades existentes (como el John Glenn College of Public Affairs, de The Ohio State University).

Esto no ha ocurrido solo con las disciplinas tradicionales, sino también con programas profesionales como, por ejemplo, administración pública, derecho, administración de empresas, trabajo social, periodismo y ciencias de la comunicación, estudios internacionales y de área, así como con estudios centrados en grupos demográficos específicos, tales como los estudios islámicos y arábigos en los Países Bajos (considérese la gran tradición de estos campos en la Universiteit Leiden) y estudios de la mujer ${ }^{13}$, estudios afroamericanos, estudios nativo-americanos y de las ciencias de la religión en Estados Unidos.

Las fronteras entre disciplinas en las ciencias naturales parecieran estar bastante claras. Sin embargo, la división entre física y química, por ejemplo, es mucho menos evidente ahora que hace cincuenta años. Las áreas de estudio en el mundo académico aún se delimitan según estructuras organizacionales del siglo XIX originadas en Alemania. En la práctica, el volumen de trabajo interdisciplinario ha tornado bastante obsoleta esa estructuración de áreas de estudio (véase Riedl 1978-1979). A su vez, los límites podrían estar bastante claros en varias de las ciencias sociales y humanidades, siempre y cuando el objeto del conocimiento de una disciplina específica realmente sea propiedad de sus investigadores. A modo de ejemplo, sería muy improbable que los teólogos se involucrasen con los objetos de conocimiento de colegas que estudian lingüística comparativa.

12 El término "generalización legaliforme" es utilizado con bastante frecuencia en las ciencias sociales. No obstante, pensándolo bien, es un poco un oxímoron: una generalización tiene las propiedades de una ley o no las tiene.

13 Los estudios de la mujer también se encuentran en las universidades neerlandesas desde la década de 1970, y la Universiteit Leiden tuvo un departamento en esta materia hasta hace poco. 
Inicialmente, el establecimiento de límites del conocimiento tenía por objeto diferenciar ciencia de lo que no es ciencia (Popper 1963). Sin embargo, desde el siglo XIX ha cobrado cada vez más mayor importancia el determinar las fronteras entre e incluso dentro de las disciplinas. Este trabajo de límites, como lo llamaba Gieryn (1983; véase también Lamont y Molnár 2002), es el instrumento mediante el cual se mantiene, refuerza, amplía y protege el conocimiento en una disciplina en particular (Good 2000: 387). Establecer límites claros resulta más fácil en algunas disciplinas que en otras.

Como se mencionó con anterioridad, la claridad de un límite alrededor de una disciplina en particular, dependerá de la medida en que su objeto de conocimiento es propiedad de su comunidad de académicos y, por lo tanto, la medida en que sus conceptos y teorías son exclusivos de ella. A modo de ejemplo, los matemáticos de todo el mundo utilizan un lenguaje universal y trabajan con mundos completamente construidos. Otro ejemplo se refiere a la comunidad de físicos, que también utilizan un lenguaje universal y trabajan en conformidad con un paradigma dominante (el modelo estándar). Observan una porción de la realidad de una manera específica y previamente acordada. Lo mismo se puede decir de químicos y biólogos. Los físicos además son quienes han llegado más cerca del ideal del conocimiento objetivo (a pesar de reconocer que nunca llegarán a alcanzarlo), es decir, a la noción de que el conocimiento existe $y$, por consiguiente, que la realidad puede ser observada con cierta independencia de la acción humana. Si las personas podemos acceder por completo a la realidad exterior (lo que Kant llamaba el objeto material) o si solo podemos observar aquella parte de la realidad que percibimos a través de nuestros sentidos (vista, olfato, tacto, oído, gusto) (Locke, Kant) y/o a través de la racionalidad (Descartes, Kant), es una pregunta que puede que nunca seamos capaces de responder ${ }^{14}$. No obstante, es vital, a lo menos, pensar en ello, pues los cientistas sociales inclinados hacia el empirismo lógico habitualmente estudian aquella parte de la realidad que es accesible por medio de conceptos y teorías (lo que Kant denominaba el objeto formal) (véase también Raadschelders y Rutgers 1989: 25) que ellos desarrollan sobre la base de una combinación de percepción sensorial y racionalidad y, cuando adoptan las ideas de Hume -de manera aún más limitada- solo aquella parte de la realidad que se refiere a hechos medibles acerca de acontecimientos, acciones y respuestas observables. En las ciencias naturales se han creado instrumentos que han ampliado en gran medida

14 Hasta donde sé, el primer académico en observar que las personas perciben cosas tanto a través del uso de sus sentidos como ("fuera de su esencia") a través de su capacidad de pensar, es Ibn Khaldûn (2005 [1967]: 333). 
nuestras capacidades sensoriales (por ejemplo, el microscopio y telescopio), pero no existen equivalentes a ello en las ciencias sociales o humanidades. Quizás las simulaciones y juegos podrían lograr algo comparable en las ciencias sociales (Heidelberg y Desai 2011), pero es probable que no puedan ampliar nuestras observaciones en la misma medida que lo han hecho en la física y astronomía el telescopio Hubble o el gran colisionador de hadrones.

Las ciencias sociales han hecho un gran esfuerzo por emular a las ciencias naturales ${ }^{15}$, impresionadas $y$, posiblemente, asombradas por los saltos revolucionarios hechos por Newton y Einstein en física, por Priestly y Lavoisier en química, por Darwin y Mendel en biología, y muchos otros. En particular, después de la Segunda Guerra Mundial, han intentado ser más científicas mediante el desarrollo de métodos cuantitativos-estadísticos y la utilización de modelos de estilo matemático. En efecto, el concepto de ciencia en las ciencias sociales pareciera ser primordialmente comprendido y aplicado en términos de metodología y métodos, con lo que, ciertamente, se ha logrado gran refinamiento y distinción. Las ciencias sociales parecen intentar y tornarse más exactas y objetivas a través de la cuantificación, tal como lo observó Van Gigch, académico en gestión de sistemas (1997: 386387, 2001a; véase también Ramos 1982: 40). Si vamos un paso más allá, el economista Von Hayek, en su discurso de aceptación del premio Nobel, planteó que lo que se considera importante en las ciencias sociales es "[...] lo que resulte ser medible. Esto se lleva en ocasiones hasta el punto de que se exige que nuestras teorías sean formuladas en términos tales que solo se refieran a magnitudes cuantificables" (1974).

No obstante, los cuantos y las matemáticas no son las única vías por la cual se ha dedicado esfuerzos sustanciales para impulsar las ciencias sociales; al parecer, la gramática y sintaxis también lo son. Es por ello que Starbuck, antiguo editor de Administrative Science Quarterly, recuerda cómo en una cátedra de ciencias sociales matemáticas dictada por Alan Newell, Herbert Simon recomendó a los estudiantes de doctorado utilizar siempre verbos en voz pasiva en sus ensayos, pues indicaba una distancia suficiente entre investigador y objeto (Starbuck 2006: 7, 40). Por lo visto, el distanciamiento impersonal se consideraba necesario para convertirse en ciencia en el sentido estricto.

Lo que imposibilita que las ciencias sociales logren ser más científicas es que su arsenal de métodos solo permite estudiar las causas materiales, es decir, se centra en el aquí y el ahora y responde preguntas acerca de

15 Con respecto a la ciencia política, véase, por ejemplo, Voegelin (1974 [1952]: 4-5). 
cómo funciona (Vanelli 2001: 53-55) ${ }^{16}$. Los científicos de las ciencias naturales, por el contrario, se centran en el estudio de las regularidades y probabilidades, y buscan comprender por qué un fenómeno natural se "comporta" de la manera en que lo hace. Se podría discutir que en las ciencias sociales también se plantean preguntas acerca del "por qué", pero a diferencia de las ciencias naturales, el consenso acerca del "por qué" de los fenómenos sociales depende en gran medida de la interpretación de cada investigador, que a su vez carece del marco nomológico (es decir, un sistema de generalizaciones relacionadas entre sí con respecto a un conjunto particular de objetos; es lo que Kuhn denominaba paradigma) que poseen las diversas disciplinas de las ciencias naturales (D'Andrade 1986: 28). En el estado actual de la física, el modelo estándar alberga todo lo que conocemos acerca del universo, pero los físicos no lo consideran ley. En efecto, han dejado de utilizar el término ley, y en cambio hablan de regularidades y de probabilidades. Ya no buscan la primera causa (es decir, la causa final o definitiva) sino que, al no estar satisfechos con el grado en que el modelo estándar les ayuda a comprender el universo, continuarán buscando una teoría unificadora.

Las consideraciones anteriores sobre ciencia, disciplina, límites, realidad y ciencias sociales, ayudan a determinar si el estudio de administración pública es una disciplina.

\section{LA ADMINISTRACIÓN PÚBLICA COMO UNA "DISCIPLINA" Y SU ENFOQUE HACIA LA INVESTIGACIÓN Y LA DOCENCIA}

¿Cómo sería el estudio de la administración pública si lograra el anhelado estatus de disciplina, es decir, ser un conjunto de conocimiento claramente delimitado de otros conjuntos de conocimiento? En materia sustantiva, se podría discutir que su objeto de conocimiento es exclusivo de ella y, es más, que sus métodos para la adquisición de conocimientos también lo son.

En lo organizacional, no cabría duda que está separada de otros estudios (por ejemplo, el derecho, la ciencia política o los estudios organizacionales,

16 Aristóteles diferenciaba las causas constitutivas o intrínsecas de aquellas activas o extrínsecas. Las primeras incluyen la causa formal (¿qué tipo de cosa es?) y la causa material (¿de qué está hecho?); las segundas se ocupan de las causas eficientes (¿cómo llegó a existir la cosa?) y las causas finales o definitivas (¿por qué llegó a existir la cosa?). 
pero podría y debería incluir estudios sobre políticas y gestión) ${ }^{17}$.

El objeto del conocimiento de la administración pública, es decir, el gobierno en sus múltiples relaciones con la sociedad, ha capturado el interés de muchos investigadores de todas las áreas de las ciencias sociales. Esto se debe a que el rápido crecimiento del gobierno (indicado anteriormente) y su penetración cada vez mayor en la sociedad, son posiblemente los fenómenos más generalizados del último siglo, y quizás más importantes que la globalización y "revolución" informativa en los últimos veinte o treinta años. Como fenómeno social, ninguna ciencia social puede darse el lujo de ignorar el impacto del gobierno sobre su interés primario de investigación (la psiquis para la psicología, los recursos limitados para la economía, la interacción humana para la sociología, el poder para las ciencia política, entre otros).

El hecho que la mayor parte de las ciencias sociales y humanidades (filosofía, teología, historia) estudien aspectos del gobierno, significa que este objeto de estudio no puede tener límites disciplinarios claros. Esto significa que aquello que constituye la calidad del conocimiento (en términos de contenidos y método) sobre el gobierno, es determinado por un grupo bastante disperso de académicos (administradores públicos, cientistas políticos, economistas, antropólogos, sociólogos, etc.). Sin embargo, no son los únicos actores que definen los contenidos y calidad del conocimiento. Los académicos tienden a evaluar la calidad en términos de rigor teórico, sofisticación metodológica y evidencia empírica, pero en el estudio de la administración pública la calidad de investigación también se evalúa en términos de conocimiento utilizable (Lindberg y Cohen 1979) y eso atañe a funcionarios de carrera, funcionarios políticos ejecutivos y legislativos, ejecutivos de empresas, grupos de presión, ciudadanos y representantes de grupos de interés. En efecto, los investigadores en administración pública no son dueños de su objeto de investigación y en este respecto no son diferentes de sus pares, por ejemplo, en derecho, medicina, enfermería, trabajo social, ingeniería o administración de empresas.

Además, y a diferencia de lo que ocurre en las ciencias naturales, no contiene paradigmas en el nivel general del estudio ni en sus diversas especializaciones, lo que impide aún más el establecimiento de límites. $\mathrm{Al}$ mismo tiempo, es importante hacer notar de que los límites que crean una

17 Nota bene, claramente la administración pública es todo esto y más; asimismo, los otros estudios arriba mencionados tampoco son disciplinas. Es, sin embargo, dudable que convertirse en disciplina sea una expectativa viable para el futuro del estudio, como se explicará más adelante. 
“disciplina” no garantizan por sí solos la calidad y utilidad del conocimiento.

Existen otros dos aspectos que impiden una identificación significativamente aceptable de límites para el estudio. En primer lugar, mientras que el gobierno es un fenómeno universal en términos de estructura, en gran medida es un fenómeno local en términos de funcionamiento (procesos y cultura). En segundo lugar, los límites del gobierno, y por ende, de su estudio, varían con el grado de intervención gubernamental en la sociedad. Así, un estudio de la administración pública en un Estado guardián tiene un ámbito mucho más limitado (por ejemplo, mantención del orden público y seguridad, prestación de servicios básicos) que otro que analiza el gobierno en un Estado de Bienestar. Además, los problemas con que tratan los gobiernos no siempre son sencillos, también pueden ser complejos e intrincados (Rittel y Webber 1973: 160). Los fenómenos naturales son definibles y separables unos de otros y pueden, por lo tanto, ser claramente definidos y delimitados con fines de investigación. Un ejemplo es suficiente para ilustrar este punto: los vulcanólogos estudian de forma específica y discreta la geología y la ciencia de una erupción; los gobiernos deben lidiar con las consecuencias de dichas erupciones, por ejemplo, para el tránsito aéreo, el transporte, la evacuación de las personas, la recuperación económica, la asistencia médica, la distribución de comida, y así sucesivamente. Lo que para el científico natural es un fenómeno que se puede reducir a un problema definible e individualizable para su análisis, resulta un problema confuso para el cientista social y el estadista, quienes no pueden seleccionar qué aspecto del problema elegir para analizar. En la administración pública, y por lo general en las ciencias sociales, la complejidad de la realidad se reduce a menudo a proporciones que permiten la observación y medición; sin embargo, ningún modelo de la realidad social (ya sea en expresión figurada ${ }^{18}$, cuantitativa o matemática) puede verdaderamente captar la realidad tan bien como la mecánica cuántica hasta ahora ha logrado captar la realidad física del universo. En lo que se refiere a los fenómenos sociales, las únicas leyes universales son bastante intrascendentes y subordinadas al contexto social (por ejemplo, si golpeamos a alguien, existe poca probabilidad que nos devuelva el golpe si

18 Habitualmente el contraste está entre el trabajo cuantitativo y cualitativo pero, mientras que el concepto de "trabajo cuantitativo" capta adecuadamente su contenido y método, el concepto de "trabajo cualitativo" es muy poco satisfactorio. Por ello, prefiero el concepto de "figuración" de Norbert Elias, que enfatiza que solo podemos comprender el mundo cuando consideramos que la realidad social es el producto de fuerzas y procesos planificados y no planificados que emanan "[...] de las formas en que las personas [son] unidas y por las presiones que ponen [...] unos a otros" (véase Elias 1987: 166, Linklater y Mennell 2010: 388). 
somos más fuertes que él o, si extendemos nuestra mano a modo de saludo, por lo general obtendremos un apretón de manos en algunas culturas, mientras que en otras podría ser que no).

Tomando como punto de partida la complejidad del objeto de investigación, Auguste Comte concluyó que las ciencias sociales eran mucho más complejas que, por ejemplo, las disciplinas puramente lógicas (las matemáticas, la física teórica) y los estudios experimentales (la física, química, biología) (Levine 1995: 164). En la misma línea, Meier argumentó que las ciencias sociales presentan un desafío mucho mayor que las ciencias naturales puesto que las primeras tienen significativos componentes de diseńo (2005: 655). También, porque abordan fenómenos que son inherentemente inestables, variables e irregulares (Kaplan 1964: 348). Es por esta razón que varios académicos han sostenido que las llamadas ciencias exactas no pueden ser aplicadas a las ciencias sociales, y sin embargo inadecuadamente lo han sido (Kaplan 1964:398, D’Andrade 1986: 39, Secord 1986: 199, Hall 1989: 33).

En general, estos estándares de ciencias naturales (reiteración, objetividad, generalización) son invocados implícitamente en el estudio de la administración pública, lo que es particularmente notorio en el debate sobre su crisis de identidad y en los juicios acerca de la calidad de su investigación. Qué implica exactamente esta crisis, no está muy claro (véase Raadschelders 1999: 287, 2011 b: 19-24) ${ }^{19}$, pero hay por lo menos tres formas diferentes en que se han sugerido soluciones.

En primer lugar, se ha definido en términos de carecer de un núcleo teórico y metodológico específico y exclusivo, y por lo general, se ve en una perspectiva de conocimiento adquirido a través de la aplicación del método científico. Un excelente ejemplo de un académico que abrazó esta postura empirista es Herbert Simon (véase nota al pie 11), quien escribió que comenzó su carrera en el remanso académico de la administración pública hace mucho tiempo con la esperanza de convertirla alguna vez en ciencia (1991: 114).

En segundo lugar, con el argumento que los valores no se pueden separar de los hechos, Waldo consideraba tres soluciones a la crisis de identidad. Consideró que la primera, la administración pública como subcampo de la ciencia política, no funcionaría puesto que el estudio aborda un contenido

19 Esto es particularmente cierto en los Estados Unidos; en la Europa continental, los investigadores en administración pública parecieran estar mucho menos preocupados de la estatura y reputación académica del estudio. 
mucho más amplio que la segunda, y porque la actitud de esta hacia la administración pública es "[...] en el mejor de los casos, de indiferencia, y a menudo de un desprecio u hostilidad no disimulados" (1968a: 8) ${ }^{20}$. Opinó que la segunda opción, considerar el estudio como una disciplina, era igualmente insatisfactoria.

Es demasiado ambicioso creer [...] que es posible identificar y desarrollar un conjunto coherente de teoría sistemática que sea sustancialmente independiente de otras ciencias sociales y solo se ocupe de la administración pública. [No es lo suficientemente ambicioso porque] Mira hacia el interior, hacia las delimitaciones conceptuales, y hacia el exterior, primordialmente hacia límites físicos. [...] Como no podemos hacer caber la amplitud y variedad de intereses actuales en una subdisciplina, tampoco podemos hacerlas caber en una disciplina (ibíd.: 9).

Lo que quedó de su enfoque fuela adopción de una perspectiva profesional "[...] sin la esperanza o la intención de convertirse en [una profesión] en ningún sentido estricto" (1968a: 9. Cursiva en el original). Al igual que la medicina, la administración pública es "[...] ciencia y arte, teoría y práctica, así como estudio y aplicación [...]” y no funciona con una teoría sino con muchos tipos de teorías (ibíd.: 10-11). Como observó Wallace Sayre, Waldo no dejó su perspectiva profesional "[...] lo suficientemente explícita para el lector" (1968: 27), y el mismo Waldo comentó más tarde, en la misma publicación, que podría haber sido mejor hablar de un "enfoque de escuela profesional" (1968: 244; énfasis en el original).

Por último, la crisis de identidad ha sido descrita por Vincent Ostrom como una consecuencia de las reformas en el gobierno y los posteriores cambios en el estudio, que condujeron, cada vez más a los Estados Unidos, a un alejamiento de las intenciones de sus fundadores, en la dirección de un gobierno centralizado y científico (1974).

Ostrom abogó por un retorno a una administración democrática basada en el autogobierno (local), caracterizada por el policentrismo y, por ende, jurisdicciones superpuestas (1974: 81, 88-89: 109). También Ventriss promovió un avance hacia el autogobierno democrático (1991: 7). A primera vista, la crisis de identidad de Ostrom podría parecer muy diferente de la identificada por Simon y Waldo. Consideremos, sin embargo, que el

20 Se puede discutir si la "hostilidad" que menciona Waldo es endémica o si se refiere al contexto temporal específico en que escribió (es decir, la ciencia política estaba en su revolución conductual). 
cambio hacia un gobierno más centralizado y científico, a contar del siglo XX, implicó un mayor énfasis en la eficiencia, estandarización y desempeño (que es la imagen tecnocrática del estudio de administración pública en los Estados Unidos) a costa de una menor atención en cuanto a los desafíos de la democracia. Por lo tanto, la identidad del estudio está fuertemente inclinada a favor de las cualidades medibles, lo que es evidente en los Estados Unidos, y que influye en la elección de métodos y enfoques que se consideran "científicos" en el sentido estricto identificado anteriormente.

El estudio de la administración pública no está solo en sus lamentos sobre una crisis de identidad. Incluso una lectura superficial de los estudios y disciplinas en las tres ramas principales del conocimiento (ciencias naturales, ciencias sociales y humanidades) revela claramente que todas las disciplinas y estudios muestran crisis de identidad, y que esto comenzó aproximadamente en la década de 1960, producto de la proliferación de especializaciones, enfoques y escuelas. Pues, sucede en este punto que la administración pública no difiere de la ciencia política, la historia, arqueología, antropología, sociología, las relaciones internacionales, la química, física, psicología, medicina, las lenguas y así sucesivamente (Raadschelders 2011b: 25-35), y podemos sumar a las matemáticas a esta lista (Kline 2010 [1980]: 260 y 371). Para aclarar este punto, las crisis de identidad producto de las especializaciones, enfoques y escuelas son un fenómeno mundial, mientras que las crisis de identidad producto de la carencia de límites parecieran ser más que nada una preocupación de la administración pública de los Estados Unidos.

Cuando se cuestiona la identidad académica de la administración pública, se hacen esfuerzos por mejorar dicha identidad y ello generalmente ocurre mediante la reflexión acerca de la calidad de su investigación y de cómo ella se puede optimizar. La investigación en administración pública ha sido etiquetada como escasamente acumulativa (Perry y Kraemer 1986: 220, Houston y Delevan 1990: 680), cléctica (Perry y Kraemer 1990: 364, Rhodes et al. 1995: 11), incapaz de satisfacer de los criterios de las corrientes tradicionales de investigación en ciencias sociales (McCurdy y Cleary 1984, White 1986, Cleary 1992, señalaron cierta mejoría), centrada en definir y conceptualizar un problema de investigación más que en desarrollar teoría (Perry y Kraemer 1986: 219, Stallings y Ferris 1988: 585, Houston Delevan 1990: 675-680), con escasa competencia para probar teorías (Perry y Kraemer 1986: 219, Stallings y Ferris 1988: 583, Rhodes et al. 1995: 11), como primordialmente descriptiva y por contener formas bastante simples de estadísticas inductivas (Rhodes et al. 
Esta revisión bibliográfica se ha centrado principalmente en revistas estadounidenses $\mathrm{y}$, en algunos casos, inglesas y australianas. Cuando nos alejamos del enfoque estadounidense del estudio y consideramos las tradiciones nacionales de la administración pública, podemos ver diferencias. Si tomamos los Países Bajos, por ejemplo, prácticamente no existe bibliografía que investigue la calidad de la investigación en administración pública en artículos y/o tesis doctorales. Tras el quincuagésimo aniversario de la revista holandesa Bestuurswetenschappen, se realizó un análisis de las tendencias tangibles, desde su creación en 1947. No pareciera que los académicos holandeses, y posiblemente tampoco sus colegas de Europa continental, sufran de una crisis de identidad comparable a aquella de sus pares estadounidenses (Raadschelders 1998b: 32).

Mientras, a principios del siglo XXI, la identidad del estudio continúa captando algo de atención (Stillman 1999, De Zwart 2002 en respuesta a Farmer 1999, Vigoda 2002, Meier y O’Toole 2007 en respuesta a Luton 2007, Raadschelders 2010). Por el contrario, la mayor parte de los trabajos que cuestionan la calidad de la investigación de la administración pública fueron publicados en las décadas de 1980 y 1990, y no está claro por qué este tipo de investigación pareciera haber estado fuera del radar durante la última década. Una explicación podría ser que, quizás, los investigadores han tomado en serio la advertencia de Hal Rainey:

Cabe preguntarse si los investigadores en administración pública podrían tener mejores avances tanto en la identidad del área [gestión pública] como en su investigación y teoría, si menos de nosotros viéramos con más detención estos temas y más de nosotros simplemente identificásemos preguntas importantes sobre la investigación teórica y trabajásemos para proporcionar respuestas a ellas (1993: 9)22.

En este enfoque existe una responsabilidad implícita de que la investigación sobre la práctica de la administración pública conducirá a la teoría y, por ende, a la identidad. Esto resulta ventajoso para el

21 La mayoría de estos hallazgos se basaron en el análisis de los contenidos de la Public Administration Review durante los ańos especificados, algunos comparados con otras revistas. El trabajo de McCurdy y Cleary se centra en la calidad de las tesis doctorales.

22 Chester Newland se refirió a estas rumiaciones como belly button pieces (comunicación por correo electrónico de David Rosenbloom al autor, 5 de abril de 2011). 
enfoque práctico característico de muchos académicos estadounidenses de administración pública, al saber que su búsqueda de conocimiento sirve para hacer un aporte positivo en forma de resultados prácticos en la vida cotidiana de las personas (Shields 2008: 211)23. Rainey no dice que los académicos deban prescindir de meditar acerca de la naturaleza de su estudio, solo afirma que las reflexiones extensas parecieran no conducir a ninguna parte.

Diecinueve años después, concuerdo con la observación de Rainey, pero cabe señalar que las reflexiones sobre lo que no se está haciendo bien en el estudio (véase las críticas mencionadas anteriormente) no nos hará avanzar en el desarrollo de una identidad académica propia. Los filósofos de la ciencia que comenzaron como físicos (por ejemplo, Paul Feyerabend, Thomas Kuhn, Stephen Toulmin), matemáticos (por ejemplo, Carl Hempel, Imre Lakatos, Bertrand Russell, Alfred North Whitehead), químicos (por ejemplo, Michael Polanyi) o psicólogos (por ejemplo, Karl Popper), consideraron importante investigar la naturaleza y significado de su conocimiento (véase Loving 1991). En particular los físicos lo han hecho porque el lenguaje milenario (en sentido literal, las palabras) disponible para describir el mundo de la mecánica cuántica probabilística ha resultado ser insuficiente. Los enormes y sorprendentes descubrimientos y consecuencias de la mecánica cuántica requirieron de cuidadosas indagaciones acerca de su significado para la ciencia y la sociedad. Si bien las ciencias sociales podrían no echar por tierra las cosmovisiones del mismo modo en que lo han hecho las ciencias naturales, deberíamos continuar cuestionando la base y el significado de nuestros hallazgos de investigación, pero hasta el momento apenas lo hemos hecho (para excepciones, Rutgers 2004, Riccucci 2010, Lee 2011, Raadschelders 2011b) y una vez que lo hagamos, debe ser por nuestros propios motivos (Ramos, véase epígrafe al inicio de este artículo). Otra explicación para el hecho que se ha prestado mucho menos atención a la calidad de la investigación en administración pública es, quizás, que los académicos consideran que las cuestiones relativas a la identidad nunca se podrán resolver de forma satisfactoria, puesto que no se puede esperar un acuerdo de si es un arte, oficio, profesión o ciencia, ni un consenso acerca de su interés central (toma de decisiones, asociación, ámbito público, interés público, gobernanza, asuntos públicos, entre otros; véase más abajo). Así, los investigadores administración pública se limitan a los cuantos y a los modelos matemáticos (Raadschelders y Lee 2011:24), aunque no en la misma medida de como ha ocurrido en la ciencia política.

23 En una nota al margen: se debería investigar sistemáticamente el cuándo y cómo la investigación de la administración pública ha marcado una diferencia en las vidas de las personas. 
Mientras que los estadounidenses son excepcionalmente sensibles a la identidad académica, y por ende estudian esto más que cualquier otro país que yo conozca, un problema mundial relacionado con la investigación es que a menudo está centrada en un área especializada, puesto que se espera que un autor muestre una familiaridad íntima con la bibliografía en su campo de interés. Es aquel nivel de especialización lo que consigue publicar artículos. Los artículos de amplio alcance que recurren a bibliografías de varias ciencias sociales tienen menos posibilidades de ser publicados pues, en primer lugar, los revisores por lo general no están familiarizados con las bibliografías fuera de su propio campo y, en segundo lugar, cuestionan con frecuencia la contribución de dichos trabajos a su área de estudio (Poteete et al. 2010: 20). En tercer lugar, no se alienta a los académicos que se están iniciando en esta actividad a realizar este tipo de trabajos, dadas las limitaciones de un sistema de nombramientos y ascensos que incentiva la cantidad de publicaciones en revistas de primer nivel (Poteete et al. 2010: 19, Nesbit et al. 2011, i24). También pareciera que los trabajos empíricos basados en evidencia y que utilizan métodos cuantitativos son percibidos como más científicos, pero sería conveniente cuestionar los problemas que surgen con la investigación empírica (Lehrer 2010) y tener presente que la epistemología guía a la metodología (Morgan 2007) y no al revés.

Con respecto a la enseñanza, los planes de estudio de administración pública existen y no pueden sino estar organizados en y para un contexto específico. En Europa existen múltiples tradiciones de administración pública (Verheijen y Connaughton 2003, Bouckaert y Van de Donk 2010), donde el plan de estudios, por lo general, se organiza en torno a cursos centrales que en gran medida están vinculados a la incubadora inicial para el estudio (por ejemplo, el derecho en Alemania y Francia; la ciencia de las organizaciones en Noruega; la ciencia política en Reino Unido). En los Países Bajos, el estudio surgió de una influencia combinada de derecho y ciencia política. También es un estudio que se percibe como bastante fragmentado, tanto en términos de contenidos como de enfoque (Kickert y Toonen 2006, Noordengraaf et al. 2006). En los Estados Unidos, el estudio se organiza en función de las especializaciones y, en efecto, los manuales o libros de texto introductorios presentan el estudio como una serie de especializaciones. Esto no es distinto hoy que en los tiempos de Siffin (1956).

Existe otra diferencia entre los diversos estudios europeos y su contraparte estadounidense, y está en la atención que se le da a la filosofía, y en particular por los temas tratados en este trabajo. Es decir, sospecho que se presta más atención a esto en Europa, y de hecho en la Universiteit Leiden, que en los Estados Unidos. Por motivos que serán aclarados en 
las próximas tres secciones, creo que es vital que cualquier secuencia de cursos a nivel de maestría y doctorado sobre métodos y estadísticas de nivel básico, intermedio y avanzado esté contenida en un curso de ontología de la existencia, epistemología de la administración, y valores en el ámbito público. Por supuesto, no existe acuerdo entre los académicos acerca de qué es en realidad la naturaleza del estudio, pero tal curso debería abordar las diferentes perspectivas sobre ello en la bibliografía (véase discusión bajo la Figura 1). En efecto, un curso donde los estudiantes se enfrenten a pensamientos sobre la ontología de la existencia y la realidad, con preguntas epistemológicas acerca de la administración pública y el gobierno, y con el rol y posición de los valores (sociales) en el discurso público, debería marcar el inicio (introducción a) y final del programa de estudio (filosofía avanzada de la administración pública) ${ }^{24}$. En el transcurso del programa, la formación en habilidades específicas y el aprendizaje de cultura cívica, que se entrega a los futuros académicos y funcionarios públicos, debería promover una perspectiva generalista acerca de la participación y posición del gobierno en la sociedad, lo que en mi opinión es algo de lo que se carece hoy en día (pero estoy dispuesto a debatir al respecto).

En la investigación y docencia, la mayor parte de la atención está centrada en el tipo de conocimiento que se puede presentar como hechos. Existen muchos cursos que presentan a la administración pública en su manifestación técnica y mecánica y, por cierto, tanto el estudio como el gobierno han llegado a ser muy buenos en la resolución de problemas sociales a partir de este ángulo técnico y mecánico. En consecuencia, y a la luz del aumento en el uso de cuantos y de las matemáticas, pareciera que las respuestas con respecto a la identidad del estudio se encuentran en la techné y la sofisticación técnica más que en la ontología, epistemología y axiología. ¿Qué camino debe tomar entonces el estudio? Regresaremos a esta pregunta en la próxima sección. Mientras tanto, y en vista del título de esta sección, ¿aún necesitamos considerar las fuentes disciplinarias e interdisciplinarias de la administración pública como un estudio? Se determinó anteriormente que la administración pública no es un

24 Se podría argumentar que las necesidades de los estudiantes de maestrías y doctorados son diferentes y, en efecto, es así como están estructurados muchos programas. Sin embargo, cabe preguntarse si acaso no facilitaría el intercambio de experiencia y conocimiento si aquellos que están en una carrera de servicio público han tenido la misma formación que aquellos que siguieron una carrera académica. Asimismo, tanto los estudiantes de maestría como los de doctorado necesitan aquella visión generalista: los primeros, porque necesitan la habilidad de ver más allá de las responsabilidades que corresponden a su propio cargo y, los segundos, porque necesitan la capacidad de evaluar el significado y consecuencias de su trabajo para la sociedad en general. 
estudio disciplinario, sino que proviene de fuentes tanto disciplinarias como interdisciplinarias. La información disciplinaria es necesaria para abordar problemas simples o definidos. Algunos buenos ejemplos son el tapado de baches en las calles y la recolección de basura. La información interdisciplinaria es necesaria para cualquier problema o desafío que trascienda una disciplina individual. Algunos ejemplos son la aprobación de nuevos medicamentos y productos alimenticios, o la decisión referente a qué hacer con la basura y desechos, determinar la planificación y demarcación para toda una ciudad, o la gestión a pequeña escala de los recursos de uso común. Estos problemas aún son relativamente sencillos pues no solo pueden ser resueltos, sino que lo pueden hacer los gobiernos $\mathrm{u}$ otros actores. La administración pública en Europa pareciera estar particularmente consciente de la interdisciplinariedad (véase Bouckaert y Van den Donk 2010); en los Estados Unidos se discute, pero existe una gran diferencia entre decir que la investigación y formación interdisciplinarias son importantes (O’Toole 1995: 296, Schroeder et al. 2004: 94) y trabajar en ello para hacerlo realidad ${ }^{25}$.

Lo que diferencia al gobierno de otros actores es su responsabilidad de lidiar con problemas difíciles o complejos que no pueden ser abordados y resueltos por ningún otro actor, puesto que el gobierno tiene la autoridad para tomar decisiones vinculantes en nombre de toda la ciudadanía. Los gobiernos tratan a diario con una gran cantidad de problemas complicados, y es por ello que la administración pública y su estudio deberían ayudar a los gobiernos en sus desafíos. En la próxima sección se discutirá cómo el estudio lo está haciendo y cómo lo podría hacer.

25 Un excelente ejemplo que me viene a la mente es el extenso trabajo realizado por Elinor Ostrom y sus múltiples colaboradores alrededor del mundo, sobre la gestión de recursos de uso común. Utilizaron, por ejemplo, tanto la teoría del juego como la descripción densa para captar, comprender e interpretar los miles de casos que ahora son archivados en el Taller de Teoría Política y Análisis de Políticas de la Universidad de Indiana (para información general, véase Toonen 2010). Su trabajo involucra múltiples métodos y utiliza fuentes interdisciplinarias. En su orientación teórica es altamente relevante para académicos con distintos historiales pero, al mismo tiempo, en su forma aplicada es útil para los gestores de recursos comunes y funcionarios de gobierno (local) (véase Ostrom 1992; Poteete et al. 2010). Si el trabajo realizado acerca de la gestión de recursos comunes es también adisciplinario, puede ser debatido, pero en general la asociación humana se convierte en un problema perverso fuera del nivel geográfico local donde los actores o participantes se conocen entre sí. Una ilustración de aquello es una publicación reciente sobre gestión ambiental (Balint et al. 2011). 


\section{LA ADMINISTRACIÓN PÚBLICA COMO UN ESTUDIO ADISCIPLINARIO: CONSIDERACIONES DESDE LA ONTOLOGÍA, EPISTEMOLOGÍA Y AXIOLOGÍA}

El hecho que el conocimiento organizado haya sido estrechamente definido como "ciencia" durante los últimos dos siglos, ha tenido en la práctica una seria consecuencia para el contenido del discurso sobre política pública, a saber, que la atención se ha centrado cada vez más en los hechos presentados por los funcionarios públicos y expertos académicos, en lugar de los valores y cómo perciben estos los expertos, funcionarios políticos y ciudadanos comunes. Está muy bien decir que los funcionarios públicos deben exponer los conflictos de valores que subyacen en casi cualquier decisión y política, como sugiere Yates (véase arriba), pero el fundamento sobre el cual se puede hacer esto es frágil en dos sentidos. En primer lugar, como observó Gawthrop, los funcionarios públicos tienen poca comprensión de los valores democráticos, éticos y morales, que son parte integral de la noción de servicio público (1998: 19). En efecto, en los programas de estudio de administración pública casi no se presta atención a la filosofía del gobierno ni a la naturaleza primordialmente moral del liderazgo ${ }^{26}$. $\mathrm{Al}$ menos en la administración pública ha aumentado la atención por la ética, pero por lo general se enseña como un curso independiente y no integrado al resto del currículum. En segundo lugar, e igualmente importante, es que en un sistema donde la supremacía de la política consagra la democracia, no se puede esperar que los funcionarios públicos expresen públicamente los conflictos de valor que se desarrollaron durante la elaboración de políticas.

Hodgkinson fue un paso más allá que Gawthrop y sostuvo que los funcionarios públicos evitan dilucidar conflictos de valores y retroceden hacia el mundo de los hechos. En sus palabras, el

\section{[...] objetivo de la burocracia [es] racionalizar y habituar los}

26 El estudio de la administración pública no está solo en esto, pues lo mismo ocurre en la administración de empresas. De hecho, el declive de los cursos de ética en los planes de estudio de administración de empresas ha generado consecuencias devastadoras para la economía y la sociedad en los Estados Unidos. Para un sorprendente y aleccionador análisis acerca de la formación estadounidense en administración de empresas desde la década de 1880, véase Khurana (2007). Es posible que se preste atención a la responsabilidad social empresarial en el sector privado, pero hablar de ello no parece haber influido en el comportamiento real. John Ralston Saul observa que los graduados de las escuelas de negocios son "adictos al poder, divorciados de cuestiones de moralidad" y que la mayoría de los profesionales están "[...] formados con una obsesión por el detalle, la acumulación de hechos y lógica interna” (1993: 22-23). 
procedimientos para la resolución de cuestiones valóricas al nivel del menor costo para la organización. La preferencia administrativogerencial por evitar "cuestiones morales" o conflictos de valores, también se puede explicar por el hecho que las resoluciones de niveles más bajos pueden ser susceptibles de compromiso y persuasión, mientras que los conflictos de niveles más altos podrían ser irreconciliables[...] (1982: 117)

Él diferenció entre tres tipos de valores (ibíd. 110-115). Los valores del Tipo I son metafísicos e incluyen, por ejemplo, los códigos éticos y mandamientos (religiosos). En sus palabras, son transracionales puesto que se basan en la creencia. Los valores del Tipo II emplean la lógica, por lo tanto son racionales. Tienen que ver con lo que es deseado (lo que es correcto) y esto se conoce como ética del deber o deontología. Para que un valor sea juzgado como correcto debe coincidir con la voluntad de la mayoría (consenso) o estar basado en una evaluación razonable de consecuencias. Por consiguiente, los valores Tipo II son eminentemente sociales por naturaleza y los ejemplos que nos entrega son el pragmatismo y utilitarismo. Finalmente, los valores del Tipo III se basan en la estructura de preferencias del individuo y, por ende, se centran en lo deseable. ¿Por quéalgo es bueno? ¿Por qué me gusta o deseo algo? A este tipo de valor lo denomina primitivo, puesto que es asocial y hedonista. Los llama subracionales y ofrece como ejemplos el hedonismo, positivismo lógico y conductismo. Resulta extraño ver el positivismo lógico y el conductismo etiquetados de esta manera, pero Hodgkinson argumenta que ambos reducen la realidad social a hechos de la naturaleza y a preferencias individuales (ibíd.: 114). Varias páginas más adelante, nos señala que las cuestiones morales pueden ser fácilmente esquivadas mediante mecanismos de evasión o de retroceso, como el gerencialismo, la impersonalidad y racionalidad burocrática, el escepticismo y positivismo. No obstante, dado que los administradores tratan a diario con valores, aún "[...] necesitan una técnica para resolver conflictos de valores que sea superior a los métodos de evitación, menor resistencia o el principio del valor mínimo" (ibíd. 146).

¿De qué manera es relevante esto para el mundo académico? En otras oportunidades he dicho que existe un lugar para el empirismo lógico en el estudio de la administración pública y que sus hallazgos pueden alimentar la formulación de políticas y, por ende, son importantes para el gobierno (Raadschelders 2011b: 174). Al mismo tiempo, hay otros mecanismos para la comprensión y no deben ser descartados como de menor valor que aquello que puede producir el estudio de hechos. No podemos sino preguntarnos si los colegas en el campo de la administración pública que se suscriben al empirismo lógico evitan explorar el significado de 
sus hallazgos más allá de las declaraciones de hechos puesto que, según Hodgkinson, está en la naturaleza del administrador evitar discusiones con carga valórica. Esta pregunta requiere más investigación empírica, al igual que la afirmación de Gawthrop acerca de la falta de comprensión de los valores democráticos ético-morales de los funcionarios públicos en el mundo occidental. También se debe examinar más las ideas de Hodgkinson puesto que, por ejemplo, su tipología de valores ha recibido variadas críticas (Evers 1985: 39-41).

La tradición empirista lógica que domina el estudio dedica poco o nada de tiempo a reflexionar acerca del significado de los hallazgos de investigación que solo se pueden basar en la lectura exhaustiva de valores y en la compresión de la teoría de valores (axiología). Los valores del sector público han sido estudiados en la administración pública (Rutgers 2010, Rutgers y Van der Meer 2010), pero necesitamos mucho más evidencia para saber hasta qué punto las consideraciones valóricas tienen preponderancia en el mundo profesional. Una forma de hacer esto es analizando cómo diferentes conjuntos de valores (por ejemplo, valores legales, de gestión, democráticos, económicos del mercado y políticos) informan, impulsan y motivan a los funcionarios públicos y con qué criterios se seleccionan ciertos valores por sobre otros (Franklin y Raadschelders 2004, Nabatchi 2011, 2012). Otra manera de hacerlo es considerando el contenido ético de las opiniones y comportamientos de los funcionarios públicos, incluyendo tanto aquellos en niveles superiores como aquellos en puestos subordinados (Yukl et al. 2011). En efecto, mientras se deja la axiología a los filósofos, el estudio de los valores es y debe ser una parte integral en la investigación y enseñanza de la administración pública, puesto que dota al estudio de un sentido del propósito (telos), de la sociedad justa, como ha seńalado Alasdair MacIntyre una y otra vez (Overeem y Tholen 2011: $727,733)$.

El reduccionismo del conocimiento hacia la "ciencia" en las ciencias sociales también ha generado una gran consecuencia en el mundo académico, tal como la orientación hacia los hechos, por un lado, y en los métodos relativos a cómo develarlos, por otro. En especial en los Estados Unidos, la formación en cualquier programa de doctorado contiene un significativo componente de métodos, y los postulantes a plazas laborales son considerados más vendibles cuando pueden demostrar sofisticación en los métodos a través de un tema de investigación en particular. En esta modalidad lógico-empirista, la realidad se da por sentada y no es digna de contemplación. Una consecuencia de ellos es que la pregunta acerca de cómo podemos saber no se hace y, menos aún, se responde. 
La pregunta ¿qué es la administración pública? contiene tanto elementos ontológicos como epistemológicos, y ha sido respondida afirmando que su objeto central es el estudio de la toma de decisiones (véase Simon 1947, Van Braam y Bemelmans-Videc 1986), la asociación (véase V. Ostrom 1974: 106), la economía política (véase Wamsley y Zald 1973), la conformación de los asuntos públicos (Ventriss 1987: 26) del Estado (véase Debbasch 1989), lo público (véase Lan y Anders 2000) o la esfera pública (véase Raadschelders 2003). Sin embargo, esta lista por sí sola es una indicación de por qué no podemos esperar integrar el estudio en torno a un único concepto central. Después de todo, ¿cómo podríamos llegar a un acuerdo respecto a qué es un concepto central? En términos más generales, quizás nunca podamos responder la pregunta ¿qué es la administración pública? a partir de su objeto de estudio (que es el gobierno en su contexto social), puesto que nunca podríamos concordar respecto a qué es en realidad ese objeto de estudio (un asunto ontológico). Tampoco puede ser respondida argumentando que el rigor de los métodos o, mejor aún, una metodología específica para el estudio (Gill y Meier 2000) consolidará su categoría académica; la metodología nunca puede definir el objeto material del conocimiento, puesto que, nuevamente, no existe consenso acerca de qué es exactamente el objeto de estudio (esto es, no puede ser determinado con el nivel de exactitud del que gozan los científicos naturales).

La respuesta a la pregunta ¿qué es la administración pública? debe basarse en consideraciones ontológicas (¿qué es la realidad?), epistemológicas (¿Qué podemos conocer?, ¿Cómo podemos conocerlo? ¿Cuáles son las fuentes de nuestro conocimiento?) y axiológicas (¿Qué valores están involucrados? ¿Cómo valoramos algo?). Se reitera la misma situación; no podemos obtener conocimientos centrándonos solo en la metodología, puesto que siempre debemos establecer primero qué puede ser medido, en otras palabras, qué se puede presentar como un "hecho". Se presta poca atención a explorar las suposiciones relativas a la naturaleza de la realidad y la existencia (la ontología) dentro de las cuales la investigación no puede sino estar contenida. Además, si bien se presta atención a los valores del sector público (un interés axiológico), esta área de investigación generalmente está separada de las múltiples investigaciones empíricas que producen correlaciones y niveles de significación sin tener en cuenta qué significan los resultados para la práctica, es decir, cómo se puede evaluar los resultados. Sin embargo, ambas son importantes, pues la ontología generará teorías acerca de qué podemos conocer (epistemología), cómo podemos producir ese conocimiento (metodología), qué prácticas de investigación aplicamos (métodos), al igual que cómo se puede valorar los hallazgos de investigación aparentemente objetivos (axiología). En la parte izquierda de la Figura 1 se grafica lo que se ha hecho en el estudio hasta 
ahora; en el lado derecho de esta figura se grafica lo que deberíamos hacer.

\section{Figura 1: La ontología, epistemología y axiología en el estudio de la administración pública}

\begin{tabular}{cc} 
Lo que hacemos & Lo que deberíamos hacer \\
\hline Ontología & Ontología \\
$\downarrow \uparrow$ & Epistemología $\leftarrow$ Axiología \\
(Epistemología) & Axiología \\
$\uparrow$ & $\downarrow$ \\
Metodología & Metodología \\
& Fuente: Elaboración propia
\end{tabular}

A modo de primer intento de aclaración y observando solo la ontología, consideremos cuatro tipos de existencia (profundizando a partir de Stout 2012: 392-393). Si creo en una existencia que es estática e inmanente, puedo conocer sobre la base de la observación, utilizando metodologías de medición (análisis de datos de encuestas, de experimentos, entrevistas, etc.). En esta visión de "la historia es eficiente" ${ }^{27}$, la necesidad de contemplar la valoración de los hallazgos es nula, puesto que los valores solo pueden afectar al mundo real con el transcurso del tiempo. Una existencia que se considera estática pero trascendente, conduciría al conocimiento a través de la fe y/o contemplación (véase Aurelius 2008). En tercer lugar, también es posible desviarse de una noción de la existencia como dinámica e inmanente, accediendo así tanto a experiencias racionales como a las falta de racionalidad, mediante la hermenéutica, fenomenología, interpretativismo, narrativismo, teoría crítica, y así sucesivamente. Por último, cuando se considera la existencia como dinámica y trascendente, lo divino y secular se fusionan por completo, aunque resulta difícil concebir una metodología para acceder a esto (piénsese en la iluminación de Buda).

27 Relacionada con la observación anterior, que la medición solo es posible cuando algo está establecido como un "hecho", la visión de que "la historia es efectiva" se captura muy bien en la siguiente cita: "Un hecho solo se convierte en tal cuando pierde toda calificación temporal y se incorpora a un gran conjunto de conocimientos elaborado por otros. Por consiguiente, existe una dificultad esencial relacionada con escribir la historia de un hecho, pues, por definición, ha perdido toda referencia histórica" (Latour y Woolgar 1986: 106). 
En la representación de Stout, son concebibles y dignas de contemplación al menos cuatro ontologías. Para simplificar, podríamos reflexionar acerca de la relación entre ontología, epistemología y axiología, comenzando por una presentación dualista, probablemente cartesiana, de la realidad. En términos generales, los cientistas sociales se pueden categorizar en dos grupos con respecto a cómo entienden la realidad. Un grupo sostiene que la realidad existe con independencia del observador y que, por ende, se puede acceder de forma objetiva. Esta posición ontológica nos conduce a una epistemología donde las hipótesis se prueban en búsqueda de una serie de leyes generalizables que son los componentes básicos de la teoría. Puesto que la realidad es independiente del observador, los valores no entran en juego en cómo se define la realidad. Ciertamente, lo que podemos conocer es producido a través de un método científico, empleando modelos matemáticos y/o métodos estadísticos cuantitativos. El conjunto específico de decisiones ontológicas, epistemológicas, axiológicas y metodológicas descrito arriba está mejor representado por el empirismo lógico y es considerado (a veces no tan ampliamente) como el más científico en el sentido estrecho de la palabra. Este empirismo lógico se basa en una ontología estática e inmanente. 


\section{Tabla 1: Características de los distintos enfoques de la investigación de la administración pública}

\begin{tabular}{|c|c|c|c|c|}
\hline $\begin{array}{c}\text { Empirismo } \\
\text { Lógico }\end{array}$ & $\begin{array}{l}\text { La realidad objetiva } \\
\text { existe, está dada y } \\
\text { es tangible }\end{array}$ & $\begin{array}{c}\text { Desarrollar hipótesis } \\
\text { comprobables; } \\
\text { generalizaciones } \\
\text { legaliformes }\end{array}$ & $\begin{array}{l}\text { Libre de valores; } \\
\text { contexto sin } \\
\text { importancia }\end{array}$ & Método científico \\
\hline Interpretativismo & $\begin{array}{l}\text { La realidad objetiva } \\
\text { no existe; la } \\
\text { realidad es relativa } \\
\text { y depende del } \\
\text { punto de vista } \\
\text { subjetivo de actores } \\
\text { independientes }\end{array}$ & $\begin{array}{l}\text { Aprender acerca } \\
\text { de los fenómenos } \\
\text { sociales; estudio de } \\
\text { lenguaje, cultura, } \\
\text { interacciones }\end{array}$ & $\begin{array}{l}\text { Hechos y valores } \\
\text { no pueden } \\
\text { ser separados; } \\
\text { atención al } \\
\text { contexto }\end{array}$ & $\begin{array}{l}\text { Investigación } \\
\text { figuracional; } \\
\text { descripción densa }\end{array}$ \\
\hline Narrativismo & $\begin{array}{l}\text { La realidad } \\
\text { objetiva no existe; } \\
\text { la "realidad" es } \\
\text { entendida a través } \\
\text { de narrativas }\end{array}$ & $\begin{array}{c}\text { Aprender acerca } \\
\text { de los fenómenos } \\
\text { sociales registrando } \\
\text { historias }\end{array}$ & $\begin{array}{l}\text { Hechos y valores } \\
\text { están unidos } \\
\text { inexorablemente; } \\
\text { no existe una } \\
\text { Verdad definitiva } \\
\text { (hecho) }\end{array}$ & $\begin{array}{c}\text { Figuracional; } \\
\text { historias en lugar } \\
\text { de cronologías }\end{array}$ \\
\hline Teoría crítica & $\begin{array}{l}\text { La realidad objetiva } \\
\text { existe, pero está } \\
\text { distorsionada por } \\
\text { nuestros valores de } \\
\text { tal forma que no } \\
\text { podemos captarla }\end{array}$ & $\begin{array}{l}\text { Tres tipos de } \\
\text { aprendizaje: } \\
\text { técnico, cognitivo y } \\
\text { emancipatorio }\end{array}$ & $\begin{array}{l}\text { La investigación } \\
\text { debe aspirar a } \\
\text { emancipar a los } \\
\text { desposeídos }\end{array}$ & $\begin{array}{c}\text { Ninguna } \\
\text { metodología en } \\
\text { concreto; sirve } \\
\text { como modo } \\
\text { para corregir } \\
\text { investigación } \\
\text { positivista/ } \\
\text { interpretativista }\end{array}$ \\
\hline Pragmatismo & $\begin{array}{l}\text { La realidad se } \\
\text { construye a través } \\
\text { de experiencias } \\
\text { transaccionales } \\
\text { de humanos en } \\
\text { un entorno en } \\
\text { constante cambio }\end{array}$ & $\begin{array}{c}\text { El aprendizaje resulta } \\
\text { de la experiencia y } \\
\text { empleo del método } \\
\text { científico }\end{array}$ & $\begin{array}{l}\text { No hay diferencia } \\
\text { fundamental } \\
\text { entre hechos y } \\
\text { valores; los valores } \\
\text { son relativos y } \\
\text { situacionales y } \\
\text { cambian cuando } \\
\text { cambia la cultura }\end{array}$ & $\begin{array}{l}\text { Diversos enfoques } \\
\text { de investigación } \\
\text { cuantitativa y } \\
\text { figuracional, } \\
\text { acentuando las } \\
\text { interacciones } \\
\text { sociales entre } \\
\text { individuos }\end{array}$ \\
\hline $\begin{array}{c}\text { Teoría de la } \\
\text { acción }\end{array}$ & $\begin{array}{l}\text { La realidad se } \\
\text { construye a través } \\
\text { del discurso moral; } \\
\text { el ser y el deber ser } \\
\text { no están separados }\end{array}$ & $\begin{array}{c}\text { El aprendizaje se } \\
\text { construye a través } \\
\text { de consideraciones } \\
\text { éticas }\end{array}$ & $\begin{array}{l}\text { No existe la } \\
\text { neutralidad del } \\
\text { investigador, ni } \\
\text { una distinción } \\
\text { entre hechos y } \\
\text { valores }\end{array}$ & $\begin{array}{l}\text { Herramientas } \\
\text { figurativas } \\
\text { para "aprender } \\
\text { haciendo"; } \\
\text { "vivir la teoría } \\
\text { en la práctica"; } \\
\text { "planificar, } \\
\text { actuar, observar, } \\
\text { reflexionar, } \\
\text { repetir" }\end{array}$ \\
\hline
\end{tabular}

Fuente: Esta tabla ha sido adaptada de otra más amplia, elaborada por James Comeaux, Lisa Frazier, Lisa Gajary, Kristin Harlow, Hyungto Hur, Olga Kondratjeva, Stephen Roll y Nicole Thomas, durante el curso "Public Affairs 890 - Logic of Inquiry", dictado por el Profesor Anand Desai durante el trimestre de invierno de 2012 en la Universidad Estatal de Ohio.

Los teóricos críticos también consideran que existe una realidad objetiva, pero que se ve distorsionada por la forma en que valoramos las cosas. Distinguen entre conocimiento técnico, cognitivo y emancipatorio; este último en particular requiere la comprensión de la medida en que los 
valores son relativos y contextuales.

El otro grupo incluye a todos aquellos que consideran que no existe una realidad objetiva, sino que esta depende del punto de vista subjetivo de actores independientes (interpretativismo), que es articulada por un narrador (narrativismo), construida a través de la interacción entre los individuos y el entorno social en que viven (pragmatismo), o construida a través del discurso moral.

En la Tabla 1 se resumen estos diversos enfoques para la comprensión del gobierno en su contexto social. La cuestión acerca de si el mundo es objetivo o construido es una "cuestión de creencias" y tiene una gran implicancia para las políticas públicas y para cómo estudiamos la administración pública. Si el mundo es objetivo, como lo sostienen los empiristas lógicos, entonces lo que sea que estudiemos no podrá ser sujeto de evaluación, puesto que los puntos de vista personales no tienen cabida en el análisis. No obstante, ni siquiera los empiristas lógicos pueden eludir los valores que inexorablemente aparecen al decidir qué y cómo medir algo. Es decir, en términos de epistemología y metodología, los valores deben ser tomados en cuenta.

\section{¿QUÉ ES LA ADMINISTRACIÓN PÚBLICA?}

Entonces preguntamos de nuevo: ¿qué es la administración pública? En primer lugar, si el estudio de la administración pública fuese considerado como una ciencia en estricto sentido, definido anteriormente, dejando de lado arte, oficio y profesión, su relevancia para el mundo real sería muy limitada. Esto no es una observación superficial, pues ha ocurrido en la ciencia política ${ }^{28}$. En cambio, la naturaleza del estudio descansa sobre un cuidadoso y delicado equilibrio de arte, oficio, profesión y ciencia. En segundo lugar, todas las ciencias sociales estudian algún aspecto u otro del gobierno en sus múltiples relaciones con la sociedad. En este sentido, la administración pública no es "dueña" de su objeto de interés del mismo modo que los físicos son dueños de los elementos a estudiar. En tercer lugar, la administración pública no solo carece de límites en torno a su

28 Existe una serie de cientistas políticos estadounidenses que ahora opinan que su estudio se ha distanciado y/o enajenado demasiado de la realidad. Para un breve resumen de sus inquietudes, véase Raadschelders (2011b: 28-29). La cuestión aquí es, por cierto, que aquello considerado "ciencia" y "método científico" por los cientistas sociales, es muy distinto de lo que consideran ciencia los científicos naturales. En palabras de Steven Weinberg, premio Nobel (física, 1979): "No tenemos un método científico fijo [...] muchos científicos tienen muy poca noción de qué es el método científico” (2001: 85), 
objeto de interés dentro del mundo académico, sino que tampoco puede evitar que otras personas, aparte de los académicos, definan cuál es y/o debería ser su objeto de interés y qué se debería hacer para ello. Por tanto, la administración pública carece en gran medida de los atributos que la podrían convertir en una disciplina en el sentido decimonónico de la palabra. Solo utiliza el conocimiento disciplinario al tratar con problemas simples y cotidianos ${ }^{29}$.

Como mínimo, el estudio es interdisciplinario. Utilizando el concepto acuñado por Whitley (1976), una disciplina tipo "paragua" (que engloba a más disciplinas). Significa que se informa, pero no deriva únicamente de las ciencias sociales, las ciencias naturales y físicas (inclusive la ingeniería) o las humanidades. La administración pública se basa en una serie de fuentes del conocimiento (Raadschelders 2011b: 76-99). La pregunta entonces pasa a ser: ¿cómo se pueden estudiar los gobiernos? La respuesta a esa pregunta revela que el estudio de la administración pública continuará enfrentándose a los desafíos de antiguas inquietudes pero debe buscar nuevas respuestas, dado que el tiempo y contexto están en constante cambio. ¿Cuáles son estos nuevos y antiguos desafíos?

En primer lugar, considerando los manuales, el estudio se puede presentar como una cadena de especializaciones, como es el caso de los Estados Unidos, o desde un punto de vista más holístico sobre la base de un meta-marco (Raadschelders 2003), como es más común en la Europa continental. El estudio en los Estados Unidos está mucho más centrado en el desarrollo de habilidades prácticas y en ser una ciencia factual, mientras que en el viejo continente existe mucho más énfasis en la Wissenschaft, centrado en desarrollar cosmovisiones y macro-perspectivas acerca del papel y posición del gobierno en la sociedad. Sin lugar a dudas, la comprensión del gobierno y la formación de (futuros) funcionarios públicos requieren un enfoque que apunte tanto a las habilidades como a las visiones de mundo.

De esto se deriva un segundo desafío: el conectar los niveles de análisis micro (individuo, grupo), meso (organización) y macro (sociedad) (Merton 1967, Luhmann 1985, Simon 1985: 303, Mouzelis 1991: 107) ya que solo esto ayudará a investigar las grandes preguntas de la sociedad actual. Esto es, no podemos suponer que los análisis de conjuntos de datos recolectados en el nivel micro proporcionen una comprensión de las tendencias en el nivel macro (falacia del nivel equivocado).

29 Véase un proyecto en el que mi colega Anand Desai y yo estamos trabajando. 
En tercer lugar, estas grandes interrogantes deben ser abordadas a través de múltiples métodos de investigación, y no limitarse a aquello que permite medición y cuantificación. Favorecer, sin discriminación, modelos cuantitativos y matemáticos, ha limitado seriamente la capacidad del gobierno para hacer frente a problemas complejos de políticas (Nabatchi et al. 2011, i34), así como la capacidad de análisis para abordarlos. ¿Qué significan las mediciones de cuántos "amigos" tienen las personas en, por ejemplo, Facebook o MySpace? ¿Qué significa saber cuán a menudo interactúan entre sí las personas, por ejemplo, por medio de tweets? ¿Comprendemos realmente la manera en que estos medios de comunicación han influenciado la naturaleza y calidad de lo que quede de interacción cara a cara y cómo esto ha influido el campo de la discusión pública? ¿Y qué impacto tienen estas comunicaciones e interacciones intensificadas, extensificadas, pero sobre todo transformadas, sobre el funcionamiento del gobierno? Para utilizar un ejemplo más claramente vinculado con el sector público: ¿Qué significa que el número de desertores de la educación secundaria sea $X$ ? ¿Qué significa que una escuela tenga éxito cuando un gran porcentaje de sus alumnos aprueban pruebas diseñadas por el estado? ¿Logran estas pruebas capturar de forma adecuada la calidad de la educación ofrecida a nuestros niños? Ciertamente, el mundo está consciente de los retos que enfrentan los niños estadounidenses de educación primaria y secundaria. No debemos ser presumidos o complacientes en relación a los retrocesos educacionales que han tenido otros países occidentales, entre ellos los Países Bajos.

Otras grandes preguntas que solo pueden ser resueltas por y a través de los gobiernos, incluyen el hambre, la pobreza, el calentamiento planetario, la diversidad social (demográfica), los conflictos religiosos y la tergiversación consciente, persistente y dolosa de las religiones del mundo, el vertimiento de desechos, los servicios de salud, por nombrar algunas ${ }^{30}$.

En cuarto lugar, las grandes interrogantes no pueden ser respondidas a través de la perspectiva o visión de mundo del especialista. La mayoría de los funcionarios públicos hoy en día terminan sus estudios universitarios y comienzan su carrera profesional como especialistas. Muchos asumirán cargos directivos de nivel medio y alto. En organizaciones que requieren de manejo de conocimientos (el gobierno sin duda es un enorme grupo de organizaciones de conocimientos), se requiere de competencias especializadas, pero lo que se espera en los niveles medio y alto, es una

30 Mientras, existe una gran interrogante epistemológica para los académicos, y es acerca del papel de la medición en la creación de conocimiento. ¿Cuáles son los límites de la medición? ¿Cuándo será insuficiente la medición para capturar la realidad? 
visión generalista sobre la organización en su entorno más importante. El estudio de la administración pública entrena y forma a los especialistas en visiones generalistas, y por ende, debe continuar encontrando "soluciones" atingentes al antiguo problema de cómo presentar esta visión generalista $y$, en el proceso, subsanar diferencias entre los que tienen un visión más profesional y los académicos.

En quinto y último lugar, todo lo anterior exige que los académicos en administración pública continúen equilibrando la investigación y docencia. Lo ideal sería que la docencia se nutra de la investigación y viceversa. Si bien se comprende que la investigación en administración pública a menudo es tan especializada como la investigación en otras disciplinas, los investigadores administración pública no deberían eludir plantearse cómo estudiar las grandes interrogantes que solo pueden ser abordadas sobre la base de fuentes de conocimiento muy diversas y empleando múltiples métodos de investigación. El conocimeinto en nuestro campo debe

[...] crecer de las tensiones, necesidades y "problemas" sociales reales [...] Cualquier problema de investigación científica que no nazca de las condiciones sociales existentes (o "prácticas") es ficticio (Dewey 1938: 499).

Una vez que los administradores públicos hagan esto, serán estos a quienes acudan los funcionarios públicos para solucionar problemas complejos. Esto nutriría inmediatamente la formación y haría frente al reto que Merriam, Simon, Waldo y Dimock plantearon a aquellos que preparan a la próxima generación de generalistas en gobierno:

Es de suponer y desear que los estudiantes del gobierno desempeñarán un papel más importante en el futuro que en el pasado, en la configuración de los tipos de educación cívica; sin embargo, esto no será posible a menos que se adquiera una visión más amplia de la relación del gobierno hacia las otras ciencias sociales, así como la función de la politica en el ámbito social (Merriam 1934: 97; énfasis en el original).

[...] la capacitación adecuada de los "administradores" no radica en el limitado campo de la teoría administrativa, sino en el amplio campo de las ciencias sociales en general (Simon 1957 [1947]: 247). [...] el pensamiento administrativo debe establecer una relación de trabajo con cada área de competencia en el ámbito del aprendizaje humano (Waldo 1984 [1948]: 203). La administración está, o al menos debería estar, ligada a materias como la filosofía, literatura, 
historia y arte, y no meramente a la ingeniería, las finanzas y la estructura (Dimock 1958: 5).

Este tipo de formación de amplio alcance aumenta el interés en la academia contemporánea, y se podría lograr mediante mayor o mejor integración de los conocimientos, de tal modo que la curiosidad de E.O. Wilson por los sintetizadores se vuelva una realidad, aunque quizás no de la forma que él espera (liderado por las ciencias naturales):

Los estudiantes con orientación profesional deberían ser ayudados a comprender que en el siglo XXI el mundo no será dirigido por aquellos que poseen mera información por sí sola. [El conocimiento] está destinado a volverse universal y democrático [...] Nos estamos ahogando en información, mientras estamos hambrientos de sabiduría. El mundo en lo sucesivo será dirigido por sintetizadores [...] (Wilson 1998: 269)

Estos cinco comentarios caracterizan el estudio de la administración pública: Algunos de utilidad a profesionales y académicos, otros interdisciplinarios, que provienen de varias fuentes del conocimiento dentro y fuera del mundo académico con el fin de avanzar en la comprensión del gobierno en la sociedad, los adisciplinarios, que lidian con problemas complejos y otros donde la pedagogía está dirigida para desarrollar la sensibilidad de los funcionarios públicos hacia las tendencias en su entorno social.

Si bien esto no es algo nuevo, aún no hemos logrado organizar nuestro estudio y currículum académico de manera adecuada.

\section{EL CAMINO A SEGUIR: UNA ADMINISTRACIÓN PÚBLICA PARA LA DEMOCRACIA TOLERANTE}

El futuro de la administración pública como estudio descansa en entregar a los funcionarios públicos, funcionarios políticos y ciudadanos una real comprensión de los complejos problemas sociales. Esto exige que complementemos e integremos a las "habilidades" (presupuestos y finanzas públicas, evaluación de programas, gestión de recursos humanos, etc.) las visiones (disciplinarias) de la civilización moderna, el desarrollo del gobierno en el transcurso del tiempo, y teorías políticas sobre la relación entre gobierno y ciudadano. Un plan de estudios de este tipo debería basarse en lo que Ramos llamaría una teoría sustantiva de la vida humana en comunidad (1981: 24-43), un concepto acuñado anteriormente por Vincent Ostrom. Así, en referencia al epígrafe de Russel al inicio de este 
trabajo, no solo deberíamos ser espartanos que se centran en formarse en métodos y habilidades, sino también atenienses que forman la mente. El estudio de la administración pública es amplio y abarca varias especializaciones. No obstante, como observó hace dieciocho años Chester Newland, editor en jefe del Public Administration Review entre 1984 y 1990, es un estudio que carece de interconexión (1994: 488). El estudio se basa en muchas fuentes del conocimiento y es, por ende, interdisciplinario por naturaleza. No obstante, si bien sus académicos han estado conectados y han utilizado otros conjuntos de conocimiento, también ha quedado en evidencia que el estudio podría mejorar mucho si se valiera de la interdisciplinariedad (Wright 2011) cuando ella sea necesaria. En efecto, y ampliando la afirmación de que la administración pública es un "campo interdisciplinario aplicado" (Hou et al. 2011, i45), la interdisciplinariedad en las soluciones ofrecidas a los problemas sociales bien podría mejorar la calidad del estudio. No obstante, el estudio también debería mejorar en el desarrollo de puntos de vista adisciplinarios cuando sea necesario, en particular al confrontar los desafíos de los grandes problemas e interrogantes mencionados arriba. Tomando en cuenta que la administración pública es un arte, oficio, profesión y ciencia, es en su calidad de interdisciplinaria y adisciplinaria, que tanto los especialistas como generalistas, académicos y profesionales, hacen mejor uso de ella. La administración pública debería prestar la misma atención a las "personas reales que hacen un trabajo real" (Box 1992: 66) que la que le presta al análisis de conjuntos de datos.

Todo esto suena más bien abstracto; de hecho, suena exactamente como lo que uno esperaría que dijese un académico. Por lo tanto, es el motivo por el cual este trabajo concluirá con un ejemplo concreto que ilustra por qué el estudio solo puede servir al gobierno y ciudadanos cuando desarrolla sus elementos históricos, ontológicos, epistemológicos y axiológicos, convirtiéndolos en el estándar en cualquier curso. Para entregar algunas líneas generales del futuro del estudio, necesitamos, una vez más, aunque de forma escueta, retroceder en el tiempo aproximadamente unos 150 años, cuando los gobiernos debieron enfrentar una gran cantidad de problemas complicados, a consecuencia de la rápida industrialización, urbanización y explosión demográfica. Es justo decir que los gobiernos y quienes los estudian lo han hecho muy bien en resolver muchos de estos problemas, pero en un nivel bastante mecánico y/o técnico. Los gobiernos han construido alcantarillados, facilitado el desarrollo de infraestructuras de transporte, estabilizado sistemas de atención de salud, desarrollado códigos de construcción y vivienda, han regulado el mercado, y así sucesivamente, de un modo que nadie hubiese imaginado hace cien o ciento cincuenta ańos. En su intento por seguir adelante y responder a las necesidades sociales, los gobiernos han abordado problemas sociales como la pobreza bajo un 
enfoque técnico, esto es, facilitando, de algún modo, una red de seguridad social, a través de subsidios de desempleo y pensiones. Por ello, la seguridad social en realidad debería llamarse seguridad económica. Evidentemente, el éxito con que los gobiernos han abordado estos y muchos otros problemas sociales y económicos varía según el país y la cultura, pero me atrevo a decir que si nuestros bisabuelos pudiesen vivenciar el papel y posición que tiene el gobierno en la sociedad hoy en día, así como la magnitud en que las políticas y servicios del gobierno contribuyen al bienestar general, quedarían asombrados.

Entonces, los principales cambios sociales y económicos de la segunda mitad del siglo XIX no siempre han sido abordados de la manera más satisfactoria, pero al menos han sido, en gran parte, resueltos. Quizás incluso podríamos decir que fue posible abordarlos porque la mayoría de las sociedades antes a la Segunda Guerra Mundial eran bastante homogéneas. Ciertamente existían diferencias políticas, pero estas se podían superar con las élites que buscaban llegar a acuerdos, como ocurría en los Países Bajos hasta la década de 1960. Si sabemos que el principal problema de las sociedades durante la segunda revolución industrial se refería a la concentración y rápido aumento demográfico (y todas sus consecuencias), así como el exceso de trabajo, ¿cuál es el mayor problema de los gobiernos actuales y por qué es aún más importante hoy en día prestar especial atención a estas consideraciones históricas, ontológicas, epistemológicas y axiológicas que en el pasado?

Permítanme sugerir que el mayor desafío que enfrentan muchos países democráticos hoy en día es el rápido aumento de diversidad social en casi cualquier lugar del mundo occidental. Las sociedades que eran relativamente homogéneas, podían acoger refugiados sin temer que cambiasen la composición sociocultural. No obstante, después de la Segunda Guerra Mundial, y en particular después de la década de 1980, los países en desarrollo, política y económicamente más desfavorecidos, han llegado en masa para acceder a la vida de abundancia del mundo occidental. Ciertamente, los Países Bajos no son una excepción (Van der Meer y Raadschelders 2010) y la tolerancia que le enorgulleció durante siglos, se ha extendido hasta el punto en que algunos buscan y consiguen dividendos políticos incitando a un miedo básico: el temor a la pérdida de identidad (nacional). Un estudio de la administración pública que incluye de forma sistemática una atención a las consideraciones históricas, ontológicas, epistemológicas y axiológicas en su investigación y enseñanza, está mucho mejor preparado para ser útil a los ciudadanos y sus gobiernos, sepultando los estereotipos y temores populistas. La narrativa dominante en el mundo occidental -con sus fundamentos en la cosmovisión judeocristiana y con 
las estructuras políticas, territoriales y organizacionales grecorromanas- es la base sobre la cual está construido gran parte del mundo occidental desde el siglo XII en adelante, y ya no es suficiente para conectar a personas con orígenes culturales, religiosos y políticos diferentes (Migdal 1997: 213, 231, Raadschelders 2002: 19-20). La influencia grecorromana, que consiste en el potencial de la democracia (Grecia) y la estructuración del gobierno por medio de administración territorial y burocratización organizacional (Roma), está muy extendida por todo el mundo. Esta parte de la narrativa occidental permanecerá por mucho tiempo, puesto que es la manera en que los gobiernos actuales buscan estructurar su funcionamiento. No obstante, el elemento judeocristiano requiere de una cuidadosa reflexión en las sociedades multiétnicas de hoy. Esta parte de la narrativa dominante está bajo presión. Para ser más precisos, se debe reanimar los austeros fundamentos de las culturas islámicas y judeocristianas, que históricamente aceptaban la diversidad dentro y entre sociedades (Siedentop 2001: 193). La mejor contribución que puede hacer el estudio de la administración pública, es dejar espacio para las consideraciones expuestas en este trabajo y cómo ellas reinforman su tradicional y práctica atención sobre los desafíos de gestión sobre economía, eficiencia y efectividad. No solo debemos recopilar datos e identificar tendencias en la búsqueda de leyes sociales, sino que también debemos considerar el bienestar social y la calidad de vida de la sociedad, al igual que las emociones y pasiones que impulsan a los seres humanos, como sugirió Commager (1950: 205). Para citar a Siedentop, ¿podemos realmente comprender "[...] el acto sexual sin ninguna referencia al amor o al deseo?” (2001: 37) ${ }^{31}$. Esto significa que los investigadores en administración pública no solo deberían ser capaces de identificar, elaborar y medir valores, sino que también explorar y contemplar las maneras de incorporarlos en el discurso y las políticas públicas. Los funcionarios políticos, al igual que aquellos que aspiran a cargos políticos, no deberían complacer al imaginario superficial, populista y estereotipado. Por el contrario, deberían tener la valentía de hablar de valores que trascienden los mantras particulares, seculares o inspirados en alguna religión, y crear plataformas en las que las personas puedan explorar los conjuntos de valores de los demás, a partir de un respeto fundamental por la humanidad en toda su diversidad. Los funcionarios públicos tienen

31 Peter Winch (1986 [1958]) se expresó en términos similares hace más de cuarenta años, esto es, que el comportamiento humano solo puede ser comprendido en un contexto social: “`sería inteligente intentar explicar cómo el amor de Romeo por Julieta se introduce en su comportamiento en los mismos términos que podríamos querer aplicar a una rata, cuya excitación sexual la hace correr a través de una rejilla con carga eléctrica para alcanzar a su compañero? ¿Acaso Shakespeare no lo hace mucho mejor?" 
el entrenamiento y la formación para alimentar las políticas públicas tanto con los hechos como con los juicios de valor utilizados en la elaboración de políticas, pero solo cuando nuestro estudio les ha dado acceso a los pensamientos y bibliografías de todo el espectro de posibilidades y puntos de vista. No cuestionaré la primacía de la doctrina política, pero la gente implícitamente sabe que los funcionarios políticos hoy en día confían en la información imparcial proporcionada por sus funcionarios públicos. Aunque Max Weber expresó preocupación por la posibilidad de que la democracia fuese asfixiada por la burocracia, cuando hablaba de la marcha inexorable de esta ${ }^{32}$, creo que su angustia con respecto a esto puede ser enterrada ${ }^{33}$. En términos generales, los funcionarios públicos $-y$ considero que ese término neutral es mucho mejor que aquel de tinte peyorativo, "burócratas"- han servido a sus funcionarios políticos de forma admirable, al igual que a los ciudadanos, de los que ellos también forman parte. Al fin y al cabo, ¿quiénes han respondido a las demandas ciudadanas durante los últimos 150 años sino los funcionarios públicos?

Un estudio de la administración pública estáverdaderamente consolidado cuando entiende que los valores desempeñan un papel importante en la sociedad multiétnica contemporánea, y por lo tanto, proporcionan una base para la formulación de políticas. Si no es así, no será útil al tipo de democracia y gobierno que trasciende las divisiones políticas, religiosas y culturales, tan comunes en las sociedades occidentales. De lo contrario, el estudio de la administración pública quedaría relegado a la oscuridad. Y, como nunca he tenido la habilidad de leer el tarot, espero que los investigadores en administración pública lo logren, tal como lo han hecho los gobiernos hasta ahora, por medio del ensayo y error.

32 Consideremos las expectativas un tanto sombrías de Weber, de la unaufhaltsame Vormarsch der Bürokratisierung en relación a su pensamiento de que la democracia sería restringida por la burocracia; véase Wie werd Demokratie auch in diesem beschränkten Sinn überhaupt möglich sein (Weber 1980: 836)

33 Simplemente consideremos el trabajo de Patrick Dunleavy (1991) que muestra cómo los funcionarios públicos han sido muy capaces de reducir la burocracia tanto en términos del tamaño del personal como en términos de regulaciones. 


\section{REFERENCIAS}

Anderson, B. (2006). Imagined Communities: Reflection on the Origin and Spread of Nationalism. Londres, Nueva York: Verso.

Aurelius, M. (2008). The Meditations of Marcus Aurelius Antoninus. Oxford: Oxford University Press.

Balint, P. J., Stewart, R. E., Desai, A. y Walters, L. C. (2011). Wicked Environmental Problems: Managing Uncertainty and Conflict. Washington: Island Press.

Bouckaert, G. y van den Donk, W. (Eds). (2010). The European Group for Public Administration (1975-2010). Perspectives for the Future. Bruselas: Bruylant.

Box, R. C. (1992). An Examination of the Debate over Research in Public Administration. Public Administration Review, 52 (1), 62-69.

Brooks, T. (2006). Plato, Hegel, and Democracy. Bulletin of the Hegel Society of Great Britain, 53-54, 24-50.

Brown, T. (2007). Coercion versus Choice: Citizen Evaluation of Public Service Quality across Methods of Consumption. Public Administration Review, 67 (3), 559-572.

Cleary, R. E. (1992). Revisiting the Doctoral Dissertation in Public Administration: An Examination of the Dissertations of 1990. Public Administration Review, 52 (1), 55-61.

Commager, H. S. (1950). The American Mind. An Interpretation of American Thought and Character Since the 1880's. New Haven: Yale University Press.

D'Andrade, R. (1986). Three Scientific World Views and the Covering Law Model. En Fiske, D. W. y Shweder, R. (Eds.), Metatheory in Social Science: Pluralism and Subjectivities. Chicago, Londres: The University of Chicago Press.

Davidson, B. (1982). The Black Man's Burden. Africa and the Curse of the Nation-State. NuevaYork: Times Books.

Debbasch,C. (1989).ScienceAdministrative;Administration Publique. Paris: Dalloz. 
Dewey, J. (1938). Logic. The Theory of Inquiry. Nueva York: Henry Holt and Company.

De Zwart, F. (2002). Administrative Practice and Rational Inquiry in Postmodern Public Administration Theory. Administration \& Society, 34 (5), 482-498.

Dimock, M. E. (1958). A Philosophy of Administration. Toward Creative Growth. Nueva York, Evanston: Harper \& Row, Publishers.

Dunleavy, P. (1991). Democracy, Bureaucracy and Public Choice. Economic Explanations in Political Science. Nueva York: Harvester Wheatsheaf.

Dyson, K. H. F. (1980). The State Tradition in Western Europe: A Study of an Idea and Institution. Oxford: Oxford University Press.

Elias, N. (1987). The Retreat of Sociologists into the Present. En Meja, V. Misgeld, D. y Stehr, N. (Eds.), Modern German Sociology. Nueva York: Columbia University Press.

Erikson, E. H. (1968). Identity: Youth and Crisis. Nueva York: W.W. Norton \& Company.

Evers, C. W. (1985). Hodgkinson on Ethics and the Philosophy of Administration. Educational Administration Quarterly, 21 (4), 27-50.

Farmer, D. J. (1999). Public Administration Discourse: A Matter of Style? Administration \& Society, 31 (3), 299-320.

Fesler, J. T. (1982). The Presence of the Administrative Past. En Fesler, J. T. (Ed.), American Public Administration: Patterns of the Past. Washington: The American Society for Public Administration.

Franklin, A. y Raadschelders, J. C. N. (2004). Ethics in Local Government Budgeting: is there a Gap between Theory and Practice? Public Administration Quarterly, 27 (4), 456-490.

Gawthrop, L. C. (1998). Public Service and Democracy. Ethical Imperatives for the $21^{\text {st }}$ Century. Nueva York, Londres: Chatham House Publishers.

Gieryn,T. F.(1983). Boundary-Workand the Demarcation ofScience from Non-Science: Strains and Interests in Professional Interests of Scientists. American Sociological Review, 48 (6), 781-795. 
Gill, J. y Meier, K. J. (2000). Public Administration Research and Practice: A Methodological Manifesto. Journal of Public Administration Research and Theory, 10 (1), 157-199.

Good, J. M. M. (2000). Disciplining Social Psychology: A Case Study of Boundary Relations in the History of the Human Sciences. Journal of the History of the Behavioral Sciences, 36 (4), 383-403.

Hall, E. T. (1989). Beyond Culture. Nueva York: Anchor Books.

Heidelberg, R. A. Desai (2011). Simulation Rules. Ponencia presentada en la conferencia anual de la American Society for Public Administration, 11-15 de abril, Baltimore, Estados Unidos.

Hodgkinson, C. (1982). Towards a Philosophy of Public Administration. Oxford: Basil Blackwell.

Hoffmann, M. C. (2002). Paradigm Lost: Public Administration at Johns Hopkins University: 1884-96. Public Administration Review, $62(1), 12-23$.

Hou, Y., Ni, A. Y., Poocharoen, O., Yang, K. y Zhao, Z. J. (2011). The Case for Public Administration with a Global Perspective. Journal of Public Administration Research \& Theory, 21 (Minnowbrook III: special issue), i45-i 51.

Houston, D.J. y Delevan, S. M. (1990). Public Administration Research: An Assessment of Journal Publications. Public Administration Review, 50 (6), 674-681.

Irvin, R.A. y Stansbury, J. (2004). Citizen Participation in Decision Making: Is It Worth the Effort? Public Administration Review, 64 (1), $55-65$.

Johnson, E. A. (1982). The Complete Administrator. Health Care Management Review, 7 (2), 73-77.

Kant, I. (1988). Logic. Nueva York: Dover. . (1996). Critique of Pure Reason. Indianapolis: Hackett.

Kaplan, A. (1964). The Conduct of Inquiry. Methodology for Behavioral Science. San Francisco: Chandler Publishing. 
Khaldûn, Ibn (2005 [1967]). The Muqaddimah. AnIntroduction to History. Princeton, Oxford: Princeton University Press.

Khurana, R. (2007). From Higher Aims to Hired Hands. The Social Transformation of American Business Schools and the Unfulfilled Promise of Management as a Profession. Princeton, Oxford: Princeton University Press.

Kickert, W. J. M. y Toonen, T. A. J. (2006). Public Administration in the Netherlands: Expansion, Diversification, and Consolidation. Public Administration, 84 (4), 969-987.

Kline, M. (2011 [1980]). Mathematics. The Loss of Certainty. New York: Fall River Press.

Kuhn, T. (1973 [1962]). The Structure of Scientific Revolutions. Chicago: The University of Chicago Press.

Lamont, M. y Molnár, V. (2002). The Study of Boundaries in the Social Sciences. Annual Review of Sociology, 28, 167-195.

Lan, Z. y Anders, K. K. (2000). A Paradigmatic View of Contemporary Public Administration Research. An Empirical Test. Administration \& Society, 32 (2), 138-65.

Lee,K. H.(2011). TheKnowledgeEvolutionofAmericanPublic Administration: A Concept, Content, and Historical Analysis of Introductory Textbooks. Tesis de doctorado, University of Oklahoma, Estados Unidos.

Lehrer, J. (2010). The Truth Wears Off. Is there something wrong with the scientific method? The New Yorker, 13 de diciembre.

Levine, D. N. (1995). Visions of the Sociological Tradition. Chicago: The University of Chicago Press.

Lindblom, C .E. (1990). Inquiry and Change. The Troubled Attempt to Understand \& Shape Society. New Haven: Yale University Press.

Lindblom, C. E. y Cohen, D. K. (1979). Usable Knowledge: Social Science and Social Problem Solving. New Haven: Yale University Press.

Linklater, A. y Menell, S. (2010). Retrospective. Norbert Elias, The Civilizing Process: Sociogenetic and Psychogenetic Investigations An Overview and Assessment. History and Theory, 49 (4), 384-411. Publicado originalmente en 1987 en Theory, Culture \& Society, 4 (2-3), 


\section{3-247.}

Loving, C. C. (1991). The Scientific Theory Profile: A Philosophy of Science Model for Science Teachers. Journal of Research in Science Teaching, 28 (9), 823-838.

Luhman, N. (1985). The Theory of Social Systems and its Epistemology: Reply to Danilo Zolo's Critical Comments. Philosophy of the Social Sciences, 16 (1), 129-134.

Luton, L. S. (2007). Deconstructing Public Administration Empiricism. Administration \& Society, 39 (4), 527-544.

Lynn, L. E. (1996). Public Management as Art, Science, and Profession. Chatham: Chatham House.

Madhi, M. (1971). Ibn Khaldûn's Philosophy of History. A Study in the Philosophic Foundation of the Science of Culture. Chicago: The University of Chicago Press.

McCurdy, H. E. y Cleary, R. E. (1984). Why Can't WeResolve the Research Issue in Public Administration? Public Administration Review, 44 (1), 49-55.

Meer, F. M. van der y Raadschelders, J. C. N. (2010). The Administration of Dutch Migration: $19^{\text {th }}$ and $20^{\text {th }}$ Centuries. En Arnold, P. (Ed.), National Approaches to the Administration of International Migration. Amsterdam, Bruselas: IOS Press/IIAS.

Meier, K. J. (2005). Public Administration and the Myth of Positivism: The AntiChrist's View. Administrative Theory \& Praxis, 27 (4), 650-668.

Meier, K. J. y O’Toole, L. J. (2007). Deconstructing Larry Luton. Or What Time is the Next Train to Reality Junction?Administration \& Society, 39 (6), 786-796.

Merriam, C. E. (1934). Civic Education in the United States. Nueva York: Charles Scribner's Sons.

Merton, R. K. (1967). On Theoretical Sociology; Five Essays, Old and New. Nueva York, Londres: The Free Press/Collier McMillan.

Migdal, J. S. (1997). Studying the State. En Lichbach, M. I. y Zuckerman, A. (Eds.), Comparative Politics. Rationality, Culture, and Sctructure. Cambridge, New York: Cambridge University Press. 
Morgan, D. L. (2007). Paradigms Lost and Pragmatism Regained. Methodological Implications of Combining Qualitative and Quantitative Methods. Journal of Mixed Methods Research, 1 (1) 4876.

Mouzelis, N. P. (1991). Back to Sociological Theory. The Construction of Social Orders. New York: St. Martin's Press.

Nabatchi, T. (2011).Value Frames in Public Administration: Looking to the Future. Ponencia presentada en la conferencia anual de la American Society for Public Administration, 11-15 de abril, Baltimore, Estados Unidos.

. (2012). Putting the "Public" Back in Public Values Research: Designing Participation to Identifyand Respond toValues. Public Administration Review, 72 (5), 699-708.

Nabatchi, T., Goerdel, H. T. y Peffer, S. (2011). Public Administration in Dark Times: Some Questions for the Future of the Field. Journal of Public Administration Research \& Theory, 21 (Minnowbrook III: special issue), i29-i43.

Nesbitt, R., Moulton, S., Robinson, S., Smith, C., DeHart-Davis, L., Feeney, M. K., Gazley, B. y Hou, Y. (2011). Wrestling with Intellectual Diversity in Public Administration: Avoiding Disconnectedness and Fragmentation while Seeking Rigor, Depth, and Relevance. Journal of Public Administration Research \& Theory, 21 (Minnowbrook III: special issue), i13-i28.

Newland, C. A. (1994). A Field of Strangers in Search of a Discipline: Separatism of Public Management Research from Public Administration. Public Administration Review, 54 (5), 486-488.

Noordegraaf, M., Bransen, T. y Huitema, D. (2006). Fragmented but Forceful: Dutch Administrative Sciences and their Institutional Evolution. Public Administration, 84 (4), 989-1006.

Ostrom, V. (1974). The Intellectual Crisis in American Public Administration. Tuscaloosa: The University of Alabama Press.

(1990). Governing the Commons. The Evolution of Institutions for Collective Action. Cambridge: Cambridge University Press. 
(1992). Crafting Institutions for Self-Governing Irrigation Systems. San Francisco: ICS Press.

O'Toole, L. J. (1995). Diversity or Cacophony? The Research Enterprise in Public Administration. Public Administration Review, 55 (3), 293297.

Overeem, P. y Tholen, B. (2011). After Managerialism: Mac Intyre's Lessons for the Study of Public Administration. Administration \& Society, 43 (7), 722-748.

Page, E. C. (2001). Governing by Numbers. Delegated Legislation and Everyday Policy-Making. Oxford, Portland: Hart Publishing.

Perry, J. L. y Kraemer, K. L. (1986). Research Methodology in the Public Administration Review: 1975-1984. Public Administration Review, 46 (2), 215-226.

Perry, J. L., K. L. Kraemer (1990). Research Methodology in Public Administration: Issues and Patterns. En. Lynn, N.B yWildavsky, A. (Eds.), Public Administration. The State of the Discipline. Chatham: Chatham House.

Phillips, D. C. (1987). Philosophy, Science and Social Inquiry. Contemporary MethodologicalControversies in Socialand Related Applied Fields of Research. Oxford: Pergamom Press.

Popper, K. (1963). Conjectures and Refutations: The Growth of Scientific Knowledge. Londres: Routledge.

Poteete, A. R., Janssen, M.A. y Ostrom, E. (2010). Working Together: Collective Action, the Commons, and Multiple Methods in Practice. Princeton: Princeton University Press.

Raadschelders, J. C .N. (1990). Plaatselijke bestuurlijke ontwikkelingen 1600-1980. Een historisch-bestuurkunding onderzoek in vier NoordHollandse gementen: Den Haag: VNG-Uitgeverij.

(1994). Understanding the Development of Local Government. Theory and Evidence from the Dutch Case. Administration \& Society, 25 (4), 410-442.

(1997). Size and Organizational Differentiation in Historical Perspective. Journal of Public Administration Research and Theory, 7 (3), 419-441. 
Brunswick: Transaction Publishers.

(1998b). Vijftig Jaar Bestuurswetenschappen: 19471996. En Jong, H. M. (Ed.), Bestuurswetenschappen. Een analyse van 50 Jaar Bestuurswetenschappen. Den Haag: VNG Uitgeverij.

. (1999). A Coherent Framework for the Study of Public Administration. Journal of Public Administration Research and Theory, 9 (2), 281-303.

(2002) An Administrative History Perspective on Church-State Relations: On the Varied Impacts of Judeo-Christian Heritage and Organized Religion. En Raadschelders, J. C .N. (Ed.), Church and State in European Administrative History. Vol.14 of the Yearbook of European Administrative History. Baden-Baden: Nomos Verlagsgesellschaft.

Armonk: M. E. Sharpe

. (2003). Government. A Public Administration Perspective.

(2008). Understanding Government: Four Intellectual Traditions in the Study of Public Administration. Public Administration, 86 (4), 925-950.

(2009) Trends in the American Study of Public Administration: What do they mean for Korean Public Administration? The Korean Journal of Policy Studies, 23 (2), 1-24.

(2010a). Identity Without Boundaries: Public Administration's Canon(s) of Integration. Administration \& Society, 42 (2), 131-159.

Detached from Historical Context? On the Nature of Time and the Need to Understand It in Government and Its Study. American Review of Public Administration, 40( 3), 235-260.

. (2011a). The Study of Public Administration in the United States. Public Administration, 89 (1), 140-155.

(2011b). Public Administration: The Interdisciplinary Study of Government. Oxford: Oxford University Press. 
Raadschelders, J. C. N. y Rutgers, M. R. (1989). Grondslagen en Grondvragen van de Bestuurskunde. Over de Methodologische Fundamenten van de Bestuurskunde. Bestuurswetenschappen, 43 (2), 72-81.

Raadschelders, J. C. N. y Lee, K. H. (2011). Trends in the Study of Public Administration: Empirical Observations and Qualitative Observations from Public Administration Review, 2000-2009. Public Administration Review, 71 (1), 19-33.

Rainey, H. G. (1993). Important Research Questions. En Bozeman, B. (Ed.), Public Management: State of the Art. San Francisco: Jossey-Bass.

Ramos, A. G.(1981). The NewScienceofOrganizations. A Reconceptualization of the Wealth of Nations. Toronto: University of Toronto Press.

Rhodes, R. A. W., Dargie, C., Melville, A. y Tutt, B. (1995). The State of Public Administration: A Professional History: 1970-1995. Public Administration, 73 (1), 1-15.

Riccucci, N. (2010). Public Administration. Traditions of Inquiry and Philosophies of Knowledge. Washington: Georgetown University Press.

Riedl, R. (1978-1979). Über die Biologie des Ursachen-Denkens: Ein evolutionistischer, systemtheoretischer Versuch. En von Ditfurth, H. (Ed.), Mannheimer Forum - Ein Panorama der Naturwissenschaften. Mannheim: Boehringer.

Rittel, H. y Webber, M. (1973). Dilemmas in a General Theory of Planning. Policy Sciences, 4 (1), 155-169.

Rugge, F. (Ed.). (2000). Administration and Crisis Management: The Case of Wartime. Bruselas: International Institute of Administrative Sciences (Cahier d'Histoire de l'Administration, no.6).

Russell, B. (1962 [1931]). The Scientific Outlook. Nueva York: W.W. Norton \& Company, Inc.

Rutgers, M. R. (2004). Grondslagen van de Bestuurskunde. Historie, Begripsvorming en Kennisintegratie. Bussum: Uitgeverij Coutinho. . (2010a). The Oath of Office as a Public Value Guardian. The American Review of Public Administration, 40 (4), 428-444. 
. (2010b). Christiaan von Wolff (1679-1754) as

Cameralistic Philosopher. Ponencia presentada en XIV IRSPM Conference, 7-9 de abril, Berne, Suiza.

Rutgers, M. R. y van der Meer, H. (2010). The Origins and Restrictions of Efficiency in Public Administration: Regaining Efficiency as the Core Value of Public Administration. Administration \& Society, 42 (7), 755779 .

Saul, J. R. (1993). Voltaire's Bastards. The Dictatorship of Reason in the West. Nueva York: Vintage Books.

Sayre, W.S. (1968) Comment on Waldo's Paper. En Charlesworth, J. C. (Ed.), Theory and Practice of Public Administration: Scope, Objectives, and Methods. Filadelfia: American Academy of Political and Social Science.

Schroeder, L. O'Leary, Jones, D. y Poocharoen, O- (2004). Routes to Scholarly Success in Public Administration: Is There a Right Path? Public Administration Review, 64 (1), 92-105.

Secord, P. F. (1986). Explanation in the Social Sciences and in Life Situations. En Fiske, D. W y Shweder, R. A. (Ed.), Metatheory in Social Science. Pluralism and Subjectivities. Chicago: The University of Chicago Press.

Shields, P. M. (2008). Rediscovering the Taproot: Is Classical Pragmatism the Route to Renew Public Administration? Public Administration Review, 68 (2), 205-221.

Siedentop, L. (2001). Democracy in Europe. Nueva York: Columbia University Press.

Siffin, W. J. (1956). The New Public Administration. Its Study in the United States. Public Administration, 34 (4), 365-376.

Simon, H. A. (1947). Administrative Behavior: A Study of DecisionMaking Processes in Administrative Organization. New York: The Free Press. . (1991). Models of My Life. New York: Basic Books.

Skowronek, S. (1982). Building a New American State. The Expansion of National Administrative Capacities: 1877- 1920. Cambridge: 
Cambridge University Press.

Stallings, R. A. y Ferris, J. M. (1988). Public Administration Research: Work in PAR: 1940-1984. Public Administration Review, 48(1), 580587.

Starbuck, W. H. (2006). The Production of Knowledge. The Challenge of Social Science Research. Oxford: Oxford University Press.

Stillman, R. J. (1999). Preface to Public Administration: A Search for Themes and Direction. Burke: Chatelaine Press.

Stout, M. (2012). Competing Ontologies: A Primer for Public Administration. Public Administration Review, 72 (3), 398-408.

Tilly, C. (1975). Reflections on the History of European State Making. En Tilly, C. (Ed.), The Formation of National States in Western Europe. Princeton: Princeton University Press.

Toonen, T. A. J. (2010). Resilience in Public Administration. The Work of Elinor and Vincent Ostrom from a Public Administration Perspective. Public Administration Review, 70 (2), 193-202.

Van Braam, A. (1989). Filosofie van der Bestuutswetenschappen. Leiden: Martinus Nijhoff.

Van Braam, A. (con M. L. Bemelmans-Videc). (1986). Leerboek Bestuurskunde. Muiderberg: Coutinho.

Van Gigch, J. P. (1997). The Design of an Epistemology for the Management Discipline Which Resolves Dilemmas AmongEthical and Other Imperatives. Systems Practice, 10 (4), 381-394.

Vanelli, R. (2001). Evolutionary Theory and Human Nature. Boston: Kluwer Academic Publishers.

Ventriss, C. (1987). Two Critical Issues of American Public Administration. Reflections of a Sympathetic Participant. Administration \& Society, 19 (1), 25-47.

(1991). Contemporary Issues in American Public Administration Education: The Search for an Educational Focus. Public Administration Review, 51 (1), 4-14.

Verheijen, T. y Connaughton, B. (Eds). (1999). Higher Education Programmes in Public Administration: Ready for the Challenge of 
Europeanisation. Limerick: University of Limerick, Occasional Paper no.3.

Verheijen, T. y Connaughton, B. (2003). Public Administration Education and Europeanization: Prospects for the Emancipation of a Discipline? Public Administration, 81 (4), 833-851.

Vigoda, E. (2002). The Legacy of Public Administration. En Vigoda, E. (Ed.), Public Administration. An Interdiscplinary Critical Analysis. Nueva York: Marcel Dekker.

Voegelin, E. (1974 [1952]). The New Science of Politics. An Introduction. Chicago: The University of Chicago Press.

Von Hayek, F. A. (1974). The Pretence of Knowledge. Nobel Memorial Prize Lecture. 11 de diciembre, Estocolmo. Disponible en http:// werdet.atspace.com/bin/hayek_lecture [06-05-2008].

Waldo, D. (1968a). Scope and Theory of Public Administration. En Charlesworth, J. C. (Ed.), Theory and Practice of Public Administration: Scope, Objectives, and Methods. Filadelfia: American Academy of Political and Social Science.

(1968b). Comment, Conference Discussion on Methodology in the Theory of Public Administration. En Charlesworth, J. C. (Ed.), Theory and Practice of Public Administration: Scope, Objectives, and Methods. Filadelfia: American Academy of Political and Social Science.

(1984 [1948]). The Administrative State. A Study of the Political Theory of American Public Administration. New York: Holmes \& Meier.

Wamsley, G. L. y Zald, M. N. (1973). The Political Economy of Public Organizations. A Critique and Approach to the Study of Public Administration. Lexington: Lexington Books.

Webber, C. y Wildavsky, A. (1986). A History of Taxation and Expenditure in the Western World. New York: Simon \& Schuster.

Weber, M. (1980). Wirtschaft und Gesellschaft. Grundriss der Verstehenden Soziologie. Tu Tübingen: J. C. B. Mohr.

Weinberg, S. (2001). Facing Up. Science and Its Cultural Adversaries. Cambridge, Londres: Harvard University Press. 
Whitley, R. D. (1976). Umbrella and Polytheistic Scientific Disciplines and Their Elites. Social Studies of Science, 6 (3- 4), 471-497.

Winch, P. (1986 [1958]). The Idea of a Social Science and Its Relation to Philosophy. London: Routledge.

Wright, B. E. (2011). Public Administration as an Interdisciplinary Field: Assessing Its Relationship with the Fields of Law, Management, and Political Science. Public Administration Review, 71 (1), 96-101.

Wunder, B. (Ed.) (1995). The Influences of the Napoleonic "Model" of Administration on the Administrative Organization of other Countries. Bruselas: International Institute of Administrative Sciences (Cahier d'Histoire de l'Administration, no.4).

Yanow, D. (1997). How Does a Policy Mean? Interpreting Policy and Organizational Action. Washington: Georgetown University Press.

Yates, D. T. (1981). Hard Choices: Justifying Bureaucratic Decisions. En Fleishman, J. L., Liebman, L. y Moore, M. H. (Eds.), Public Duties: The Moral Obligations of Government Officials. Cambridge: Harvard University Press.

Yukl, G., Mahsud, R., Hassan, S., y Prussia, G. E. (2011). An Improved Measure of Ethical Leadership. Journal of Leadership and Organizational Studies. Disponible en línea el 11 de diciembre de 2011.

Recibido: 20-05-2015

Aceptación de la versión final: 02-06-2016 